\title{
DETERMINATION OF JOIN REGIONS BETWEEN CARBON NANOSTRUCTURES USING VARIATIONAL CALCULUS
}

\author{
D. BAOWAN ${ }^{1}$, B. J. COX ${ }^{\bowtie 2}$ and J. M. HILL ${ }^{2}$
}

(Received 15 January, 2013; revised 11 April, 2013)

\begin{abstract}
We review the work of the present authors to employ variational calculus to formulate continuous models for the connections between various carbon nanostructures. In formulating such a variational principle, there is some evidence that carbon nanotubes deform as in perfect elasticity, and rather like the elastica, and therefore we seek to minimize the elastic energy. The calculus of variations is utilized to minimize the curvature subject to a length constraint, to obtain an Euler-Lagrange equation, which determines the connection between two carbon nanostructures. Moreover, a numerical solution is proposed to determine the geometric parameters for the connected structures. Throughout this review, we assume that the defects on the nanostructures are axially symmetric and that the into-the-plane curvature is small in comparison to that in the twodimensional plane, so that the problems can be considered in the two-dimensional plane. Since the curvature can be both positive and negative, depending on the gap between the two nanostructures, two distinct cases are examined, which are subsequently shown to smoothly connect to each other.
\end{abstract}

2010 Mathematics subject classification: primary 49K15; secondary 51P05, 92E10.

Keywords and phrases: variational calculus, nanostructures, carbon nanotubes, $\mathrm{C}_{60}$ fullerenes, geometry.

\section{Introduction}

Carbon nanostructures such as carbon nanotubes and $\mathrm{C}_{60}$ fullerenes have received much attention for their applications in future nanoscale devices, since they exhibit many advantageous physical and electronic properties. Many carbon nanostructures are based on the hexagonal graphene sheet. In particular, single-walled carbon nanotubes can be thought of as a graphene sheet which is rolled up to form a cylinder, and multi-walled carbon nanotubes can be envisaged as multilayers of graphene

This is a contribution to the series of invited papers by past ANZIAM medallists (Editorial, issue 52(1)). J. M. Hill was awarded the 2008 ANZIAM medal.

${ }^{1}$ Department of Mathematics, Faculty of Science, Mahidol University, Rama VI Rd., Bangkok 10400, Thailand; e-mail: duangkamon.bao@mahidol.ac.th.

${ }^{2}$ Nanomechanics Group, School of Mathematical Sciences, The University of Adelaide, South Australia 5005, Australia; e-mail: barry.cox@ adelaide.edu.au,jim.hill@ adelaide.edu.au.

(C) Australian Mathematical Society 2013, Serial-fee code 1446-1811/2013 \$16.00 
sheets which are rolled up coaxially. Moreover, single-walled carbon nanotubes have received much attention because of their unique electronic and mechanical properties [11]. In this paper, we review a particular variational technique to determine the join region between such carbon nanostructures. This review constitutes a summary of the present authors' work in this area, and deals with a continuous approximation to an essentially complicated discrete joining problem. Previous joining problems are based on matching hexagonal and pentagonal structures such that Euler's theorem for polyhedra is correctly satisfied [18, pp. 115-135]. Here we adopt an entirely novel and simple continuous variational approach to these problems.

Although classical applied mathematical modelling has been widely used for solving problems in many areas, it has not yet been widely exploited in the field of nanotechnology, which tends to be dominated by molecular dynamics simulations, and by experimentation to a lesser extent. This review utilizes an applied mathematical approach to model the join region between two connecting nanostructures. The successful design of many novel nanoelectronic devices will require a thorough understanding of the geometric joining issues between nanostructures. In this review, we employ classical variational calculus to formulate a continuous approximation to the essentially discrete problem of determining the join region between two carbon nanostructures. In formulating this approach, there is some evidence that carbon nanotubes deform as in perfect elasticity, and rather like the elastica [23], and therefore we seek to minimize the elastic energy, but we take into account that the join region involves a finite number of discrete bonds. Here we employ the calculus of variations to minimize the curvature subject to a length constraint, to obtain an Euler-Lagrange equation, which determines the connection between two carbon nanostructures. Furthermore, we present a numerical solution to determine values of the geometric parameters for the connected structures. Throughout this review, the various defects on the nanostructures are assumed to be axially symmetric, so that the problem can be considered in the two-dimensional plane. Depending on the gap between the two nanostructures, the curvature can be both positive and negative, and therefore two distinct models are examined, which we later show to continuously connect to each other. The five joining problems considered here are as follows:

- two carbon nanotubes of differing radii [5];

- a carbon nanotube and a flat graphene sheet [9];

- a carbon nanotube and a $\mathrm{C}_{60}$ fullerene [3];

- a carbon nanotube and a carbon nanocone [4];

- two fullerenes of differing radii [2].

Note that we also assume here that the curvature in the two-dimensional plane provides the major contribution to the elastic energy, and we ignore any elastic energy arising from the into-the-plane curvature. In effect, we are assuming that the major stretching of the linkages occurs in the two-dimensional plane, rather than the intothe-plane cross linking. This may be justified by an examination of the discrete joining methods such as the least squares approach by the present authors [1]. 


\section{Calculus of variations}

This section gives the basic equations of the calculus of variations, which we use to determine the curve adopted by a line connecting two carbon nanostructures. In the terminology of the calculus of variations, we seek to determine the curve $y=y(x)$, with element of arc length $d s$, which minimizes the functional $J[y]$ given by

$$
J[y]=\int_{0}^{\ell} \kappa^{2} d s+\lambda \int_{0}^{\ell} d s,
$$

where $\kappa$ is the curvature, $\lambda$ is a Lagrange multiplier corresponding to the fixed length constraint, $\ell$ is the prescribed length of the join, and we denote the boundaries of the join region by $x_{0}$ and $x_{1}$, such that at $x=x_{0}$ we have $s=0$ and at $x=x_{1}$ we have $s=\ell$. For a two-dimensional curve $y=y(x)$,

$$
\kappa=\frac{y^{\prime \prime}}{\left(1+y^{\prime 2}\right)^{3 / 2}}, \quad d s=\left(1+y^{\prime 2}\right)^{1 / 2} d x,
$$

and hence

$$
J[y]=\int_{x_{0}}^{x_{1}}\left(\frac{y^{\prime \prime 2}}{\left(1+y^{\prime 2}\right)^{5 / 2}}+\lambda\left(1+y^{\prime 2}\right)^{1 / 2}\right) d x,
$$

where primes throughout denote differentiation with respect to $x$. On applying the delta variational operator and integrating by parts twice, we derive in the usual way the equation

$$
\delta J[y]=\left[\left(F_{y^{\prime}}-\frac{d}{d x} F_{y^{\prime \prime}}\right) \delta y+F_{y^{\prime \prime}} \delta y^{\prime}\right]_{x_{0}}^{x_{1}}+\int_{x_{0}}^{x_{1}}\left(F_{y}-\frac{d}{d x} F_{y^{\prime}}+\frac{d^{2}}{d x^{2}} F_{y^{\prime \prime}}\right) \delta y d x,
$$

where subscripts denote partial derivatives and $F$ is given by

$$
F\left(y^{\prime}, y^{\prime \prime}\right)=\frac{y^{\prime \prime 2}}{\left(1+y^{\prime 2}\right)^{5 / 2}}+\lambda\left(1+y^{\prime 2}\right)^{1 / 2} .
$$

For the present problems, we require the natural or alternative boundary condition which applies when the $y$-coordinate at the $x=x_{1}$ boundary is not prescribed, and which is derived from the first term in (2.2) and given by

$$
\left[F_{y^{\prime}}-\frac{d}{d x} F_{y^{\prime \prime}}\right]_{x=x_{1}}=0 .
$$

The usual Euler-Lagrange equation is obtained from the integral term in (2.2) and can be written as

$$
F_{y}-\frac{d}{d x} F_{y^{\prime}}+\frac{d^{2}}{d x^{2}} F_{y^{\prime \prime}}=0 .
$$

On using the above two equations, and performing one integration, since $F$ is independent of $y$, and setting the constant to zero, after a further integration we deduce

$$
F-y^{\prime \prime} F_{y^{\prime \prime}}=-\alpha,
$$




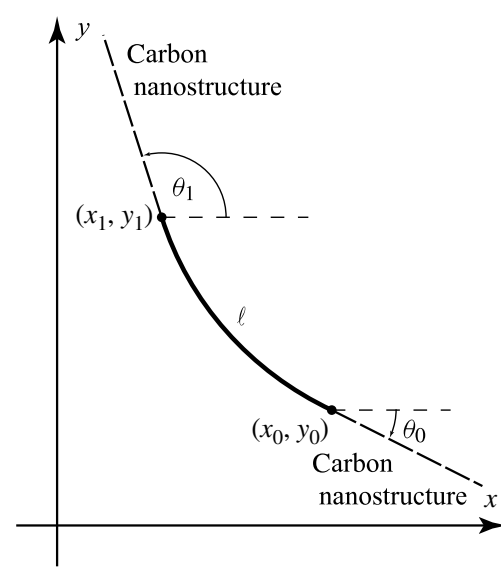

Model I

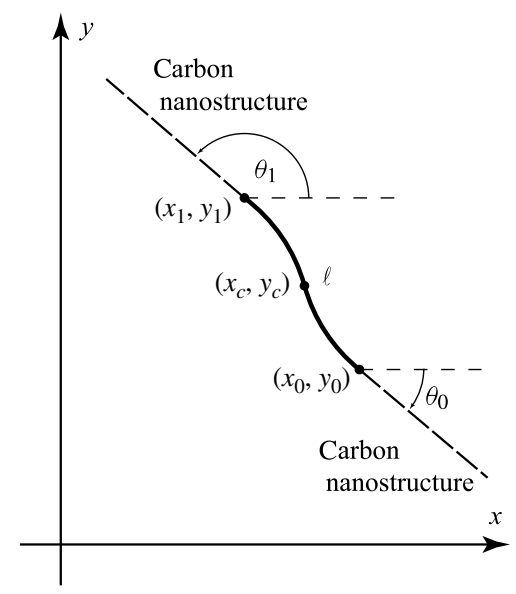

Model II

FIGURE 1. Model I, curvature always positive; and Model II, curvature both positive and negative.

where $\alpha$ is an arbitrary constant of integration. We now substitute (2.3) into (2.4), from which we obtain

$$
\kappa^{2}=\frac{y^{\prime \prime 2}}{\left(1+y^{\prime 2}\right)^{3}}=\lambda+\frac{\alpha}{\left(1+y^{\prime 2}\right)^{1 / 2}},
$$

so that the curvature $\kappa$ is given by

$$
\kappa= \pm\left(\lambda+\frac{\alpha}{\left(1+y^{\prime 2}\right)^{1 / 2}}\right)^{1 / 2} .
$$

\section{Determining the join region}

In this section we present a general formulation for all the subsequent specific problems, and we have in mind a connection beginning at a prescribed starting boundary location $\left(x_{0}, y_{0}\right)$ such that the boundary makes an angle $\theta_{0}$ with the positive direction of $x$. The join region then goes to the second boundary point, denoted by $\left(x_{1}, y_{1}\right)$, where $x_{1}$ and the angle $\theta_{1}$ made with the positive direction of the $x$-axis are prescribed, but $y_{1}$ is determined from the natural boundary condition as mentioned in the previous section. In the following analysis the sign of the curvature $\kappa$ is the key issue, and we consider two models. As indicated in Figure 1, the first model has no change in the sign of the curvature, and in the second model the curvature changes sign in the join region. In this general description we assume that the curvature is positive for Model I, and for Model II we assume that the curvature is positive adjacent to $\left(x_{0}, y_{0}\right)$ and becomes negative before reaching $\left(x_{1}, y_{1}\right)$. However, we note that the opposite of these assumptions can just as easily be used and the resulting mathematics varies only by a change of sign in the explicit formulae for $x$ and $y$, as is seen subsequently. 
3.1. Model I: curvature always positive On assuming that the curvature is always positive and making the substitution $y^{\prime}=\tan \theta$, equation (2.5) becomes

$$
\kappa=(\lambda+\alpha \cos \theta)^{1 / 2},
$$

and from the definition of curvature (2.1), and making the same substitution for $y^{\prime}$, we deduce that

$$
\frac{d y}{d \theta}=\frac{\sin \theta}{(\lambda+\alpha \cos \theta)^{1 / 2}} .
$$

To simplify some of the formulae, we now introduce a new parametric variable $\phi$ as follows:

$$
\cos \theta=1-2 k^{2} \sin ^{2} \phi
$$

where $k=[(\lambda+\alpha) /(2 \alpha)]^{1 / 2}$. We deduce

$$
\frac{d y}{d \phi}=2 \beta k \sin \phi
$$

where $\beta=(2 / \alpha)^{1 / 2}$. Upon integrating the above equation and using the boundary condition at the point $\left(x_{0}, y_{0}\right)$,

$$
y(\phi)=y_{0}+2 \beta k\left(\cos \phi_{0}-\cos \phi\right), \quad \phi_{0}=\sin ^{-1}\left(\frac{1-\cos \theta_{0}}{2 k^{2}}\right)^{1 / 2} .
$$

By precisely the same method we derive

$$
\frac{d x}{d \theta}=\frac{\cos \theta}{(\lambda+\alpha \cos \theta)^{1 / 2}} .
$$

On transforming to the new parametric variable $\phi$,

$$
\frac{d x}{d \phi}=\beta \frac{\left(1-2 k^{2} \sin ^{2} \phi\right)}{\left(1-k^{2} \sin ^{2} \phi\right)^{1 / 2}}=\beta\left[2\left(1-k^{2} \sin ^{2} \phi\right)^{1 / 2}-\left(1-k^{2} \sin ^{2} \phi\right)^{-1 / 2}\right],
$$

which upon integrating gives

$$
x(\phi)=x_{0}+\beta\left\{2\left[E(\phi, k)-E\left(\phi_{0}, k\right)\right]-\left[F(\phi, k)-F\left(\phi_{0}, k\right)\right]\right\},
$$

where $F(\phi, k)$ and $E(\phi, k)$ denote the usual Legendre incomplete elliptic integrals of the first and second kinds, respectively, as defined by Byrd and Friedman [6]. Noting that $\phi_{1}=\sin ^{-1}\left[\left(1-\cos \theta_{1}\right) /\left(2 k^{2}\right)\right]^{1 / 2}$, and using (3.3) and (3.4), we deduce that

$$
\begin{aligned}
& x_{1}=x_{0}+\beta\left\{2\left[E\left(\phi_{1}, k\right)-E\left(\phi_{0}, k\right)\right]-\left[F\left(\phi_{1}, k\right)-F\left(\phi_{0}, k\right)\right]\right\}, \\
& y_{1}=y_{0}+2 \beta k\left(\cos \phi_{0}-\cos \phi_{1}\right) .
\end{aligned}
$$

Considering the arc length $\ell$, which is by definition

$$
\ell=\int_{x_{0}}^{x_{1}}\left(1+y^{2}\right)^{1 / 2} d x
$$


and making the substitution to the variable $\phi$ and integrating,

$$
\ell=\beta\left[F\left(\phi_{1}, k\right)-F\left(\phi_{0}, k\right)\right] .
$$

Now we define a dimensionless parameter $\mu=\left(x_{1}-x_{0}\right) / \ell$, which is given by

$$
\mu=2\left(\frac{E\left(\phi_{1}, k\right)-E\left(\phi_{0}, k\right)}{F\left(\phi_{1}, k\right)-F\left(\phi_{0}, k\right)}\right)-1 .
$$

Thus, for prescribed values of $x_{0}, x_{1}$ and $\ell$, equation (3.7) can be numerically solved to determine the relevant value of $k$. By substitution of $k$ into (3.6), the value of $\beta$ may be determined and therefore $y_{1}$ may be obtained from (3.5).

3.2. Model II: curvature both positive and negative In this model, we use $\left(x_{c}, y_{c}\right)$ to denote the point at which the curvature changes sign from positive to negative. This can be found by solving (3.1) for $\kappa=0$, giving $\theta_{c}=\cos ^{-1}(-\lambda / \alpha)$, and upon making the substitution to $\phi$ given in (3.2), we find that $\phi_{c}=\pi / 2$. Thus substituting this value into (3.3) and (3.4),

$$
\begin{aligned}
& x_{c}=x_{0}+\beta\left\{2\left[E(k)-E\left(\phi_{0}, k\right)\right]-\left[K(k)-F\left(\phi_{0}, k\right)\right]\right\}, \\
& y_{c}=y_{0}+2 \beta k \cos \phi_{0},
\end{aligned}
$$

where $K(k)$ and $E(k)$ denote the complete elliptic integrals of the first and second kinds, respectively. In the join region between $\left(x_{c}, y_{c}\right)$ and $\left(x_{1}, y_{1}\right)$ we take the negative sign from (2.5), and by following the same method as in Model I, we derive

$$
\frac{d x}{d \phi}=-\beta \frac{\left(1-2 k^{2} \sin ^{2} \phi\right)}{\left(1-k^{2} \sin ^{2} \phi\right)^{1 / 2}}, \quad \frac{d y}{d \phi}=-2 \beta k \sin \phi .
$$

Thus, in this second region,

$$
\begin{aligned}
& x(\phi)=x_{c}+\beta\{2[E(k)-E(\phi, k)]-[K(k)-F(\phi, k)]\}, \\
& y(\phi)=y_{c}+2 \beta k \cos \phi,
\end{aligned}
$$

and hence the second boundary is given by

$$
\begin{aligned}
& x_{1}=x_{0}+\beta\left\{2\left[2 E(k)-E\left(\phi_{0}, k\right)-E\left(\phi_{1}, k\right)\right]-\left[2 K(k)-F\left(\phi_{0}, k\right)-F\left(\phi_{1}, k\right)\right]\right\}, \\
& y_{1}=y_{0}+2 \beta k\left(\cos \phi_{0}+\cos \phi_{1}\right) .
\end{aligned}
$$

We note that in this second region we have changed the sense of the variable $\phi$ with respect to the direction of the joining line, and that therefore the arc length, determined in two parts, is given by

$$
\begin{aligned}
\ell & =\int_{x_{0}}^{x_{c}}\left(1+y^{\prime 2}\right)^{1 / 2} d x+\int_{x_{c}}^{x_{1}}\left(1+y^{\prime 2}\right)^{1 / 2} d x \\
& =\beta \int_{\phi_{0}}^{\pi / 2}\left(1-k^{2} \sin ^{2} \phi\right)^{-1 / 2} d \phi+\beta \int_{\phi_{1}}^{\pi / 2}\left(1-k^{2} \sin ^{2} \phi\right)^{-1 / 2} d \phi \\
& =\beta\left[2 K(k)-F\left(\phi_{0}, k\right)-F\left(\phi_{1}, k\right)\right] .
\end{aligned}
$$




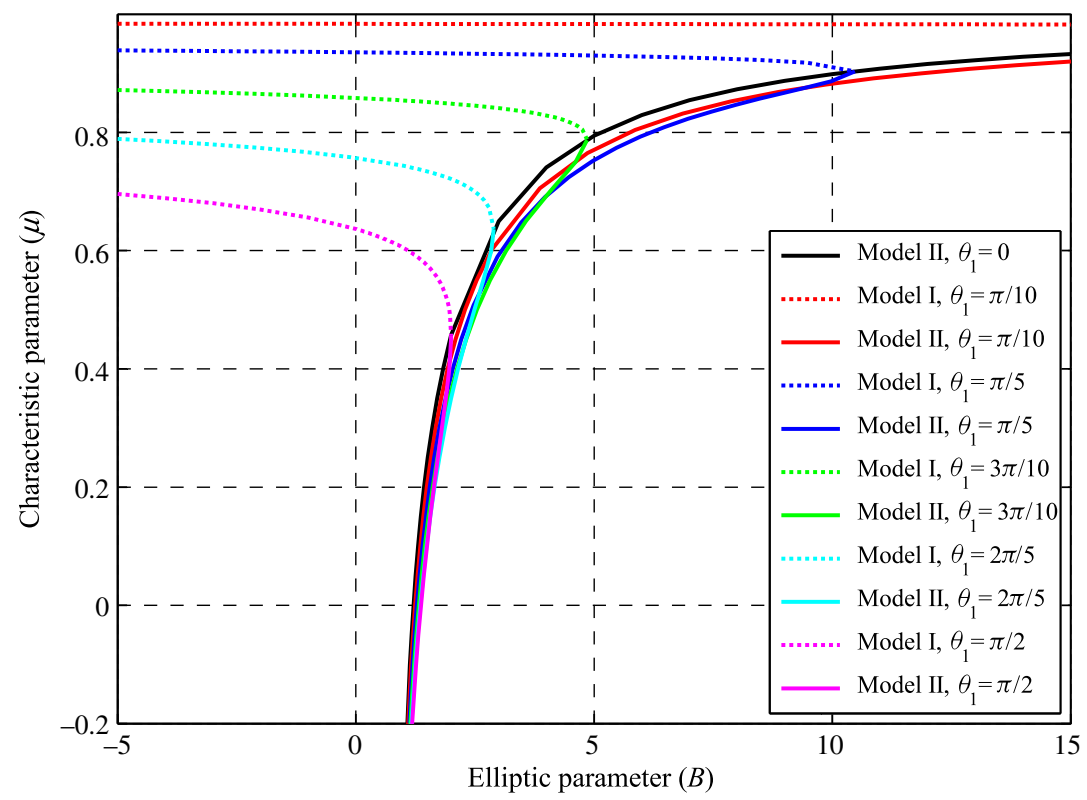

FIGURE 2. Plot of the characteristic parameter $\mu$ for various values of the elliptic parameter $B$ for $\theta_{0}=0$ and $\theta_{1}$ as indicated in the legend. (Colour available online.)

As before, we define a dimensionless parameter $\mu=\left(x_{1}-x_{0}\right) / \ell$, which for Model II is given by

$$
\mu=2\left(\frac{2 E(k)-E\left(\phi_{0}, k\right)-E\left(\phi_{1}, k\right)}{2 K(k)-F\left(\phi_{0}, k\right)-F\left(\phi_{1}, k\right)}\right)-1 .
$$

Again, for prescribed values of $x_{0}, x_{1}$ and $\ell$, equation (3.10) can be numerically solved to determine the value of $k$. Then by substitution of $k$ into (3.9), the value of $\beta$ may be determined and therefore $y_{1}$ can be obtained from (3.8).

3.3. The characteristic parameter $\boldsymbol{\mu}$ In this section we examine the numerical solution of (3.7) and (3.10), both of which are characterized by the nondimensional parameter $\mu=\left(x_{1}-x_{0}\right) / \ell$ and subject to the constraint $-1<\mu<1$. Figure 2 shows the relation between the parameter $\mu$ and a new variable $B=1 / k^{2}$. In this figure, the angle at the beginning of the join region is $\theta_{0}=0$, and we plot the relation between $\mu$ and $B$ for various values of $\theta_{1}$. We see that except for the case where $\theta_{1}=0$, all curves have a critical value of $\mu=\mu_{1}$. For $\mu>\mu_{1}$ Model I applies, and for $\mu<\mu_{1}$ Model II applies. In the special case of $\theta_{1}=0$, it is apparent that $\mu_{1}=1$.

In Figure 3 we plot the relation between $\mu$ and $B$, where in this case we have fixed the angle at the end of the joint region, $\theta_{1}=\pi / 2$, and show curves for a range of values for $\theta_{0}$. In this analysis we see that there are several critical values of $\mu$. Firstly, there are the values $\mu_{1}$ and $\mu_{2}$, where Model II applies for $\mu<\mu_{1}$ and $\mu>\mu_{2}$, and Model I applies in the range $\mu_{1}<\mu<\mu_{2}$. Secondly, in all cases except for the special case 


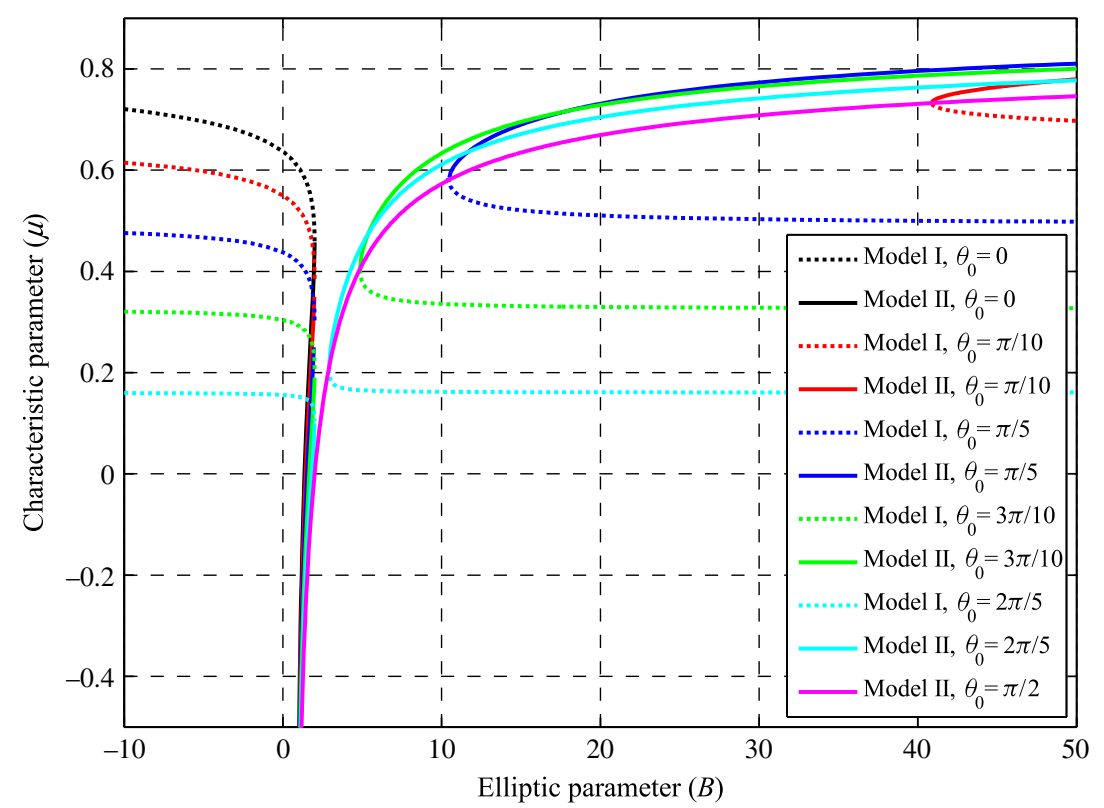

FIGURE 3. Plot of the characteristic parameter $\mu$ for various values of the elliptic parameter $B$ for $\theta_{1}=\pi / 2$ and $\theta_{0}$ as indicated in the legend. (Colour available online.)

of $\theta_{0}=0$, we also see that there is a discontinuity in the Model I part of the curve where it jumps from $-\infty$ to $\infty$. We denote this point of discontinuity with $\mu=\mu_{0}$, and note that for all the curves shown in Figure 3, when $\mu_{1}<\mu<\mu_{0}$ they apply for $B<2$, and if $\mu_{0}<\mu<\mu_{2}$ then the curve lies strictly in the range $B>2$.

Finally, we briefly comment on another critical value of $\mu=\mu_{3}$, which occurs at $B=0$. In both Figures 2 and 3 we see that crossing the $\mu$-axis, when it does occur, happens exclusively in the Model I part of the curve. The reason for this is that $B=0$ is the special case where the elliptic functions degenerate into the standard trigonometric functions and the join region is simply a circular arc. Since a circular arc has no change in the sign of the curvature, $\mu_{3}$ must occur in the Model I region of the curve, and furthermore, since it occurs in the range $B<2$ it must occur in the region $\mu_{1}<\mu_{3}<\mu_{0}$.

\section{Application to various physically motivated situations}

4.1. Two nanotubes of differing radii The principle of multiple electronic devices is based on a junction of one metallic and one semiconducting material. At the nano scale, single-walled carbon nanotubes can behave either as a metal or a semiconductor, depending on the molecular structure. Accordingly, understanding and modelling the join between any two carbon nanotubes with different electronic properties, metallic and semiconductor, is necessary in order to create new novel nanodevices. The electronic structure of carbon nanotubes is determined by two integers $(n, m)$ which define the chiral vector $\mathbf{C}=n \mathbf{a}_{1}+m \mathbf{a}_{2}$, where $\mathbf{a}_{1}$ and $\mathbf{a}_{2}$ are basis vectors. If $(n, m)$ 


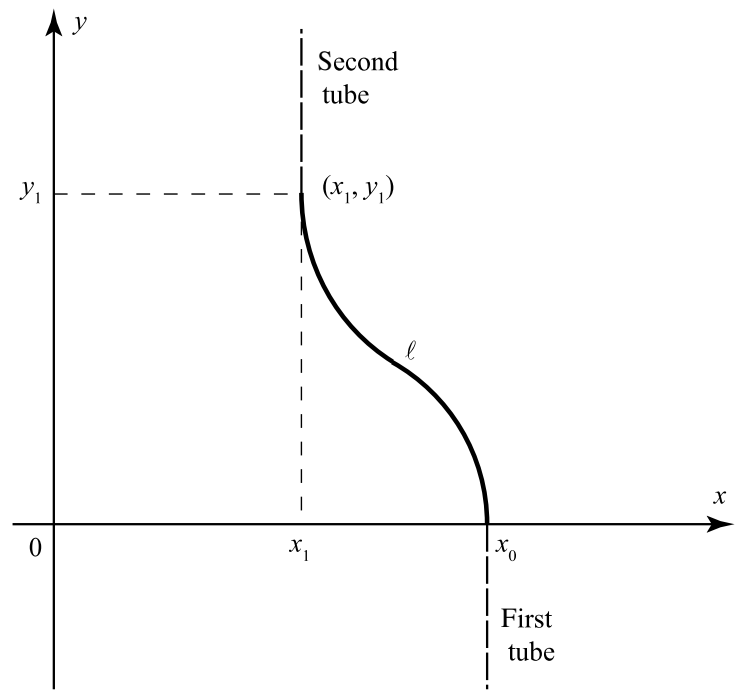

FIGURE 4. Geometry of the model for joining two nanotubes which has positive and negative curvatures in the join region.

is a multiple of 3, the tube is metallic; otherwise, the tube is a semiconductor. Moreover, these integers $(n, m)$ relate to the radius of the carbon nanotube through the expression $\sigma \sqrt{3\left(n^{2}+n m+m^{2}\right)} / 2 \pi$, where $\sigma$ represents the carbon-carbon bond length [12]. Saito et al. [17] proposed a model for the join structure of two carbon nanotubes based on a projection method, which depends only on the integers $(n, m)$, and the incorporation of a single pentagonal ring. In this section, we model the join structure for any two nanotubes by taking into account only their radii. We adopt the calculus of variations formulation to determine the join curve between the two nanotubes as shown in Figure 3.

In this case, the angles satisfy $\theta_{0}=\theta_{1}=\pi / 2$, which, as can been seen from Figure 4 , leads to a curve comprising of entirely Model II behaviour. This is not unexpected: since the two nanotube walls are parallel, the join region must curve from the starting point in the direction of the second tube and then undergo a sign change in the curvature to smoothly join at the end point. The solution, which is characterized by the nondimensional parameter $\mu=\left(x_{1}-x_{0}\right) / \ell$ subject to the constraint $-1<\mu<1$, is examined in further detail. In Figure 5 we show the relationship between the parameters $\mu$ and $B$, and this relationship can be divided into two regions. The first region is $-1<\mu<0$, which corresponds to $B<2$. In this region, $\alpha$ is always positive and the modulus $k$ is strictly real. The second region is $0<\mu<1$, which corresponds to $B>2$. The value of $B$ is always positive, and it corresponds to a negative value of $\alpha$ and a complex value of the angle $\phi$ of the form $-\pi / 2+i \varphi$. This region applies where $y^{\prime}\left(x_{0}\right)=y^{\prime}\left(x_{1}\right)=\infty$, and where $x_{0}<x_{1}$.

Following the work of Saito et al. [17], we plot the join regions $y(x)$ between two carbon nanotubes $(12,0)-(8,0)$ and $(12,0)-(9,0)$ as illustrated in Figure 6. Assuming 


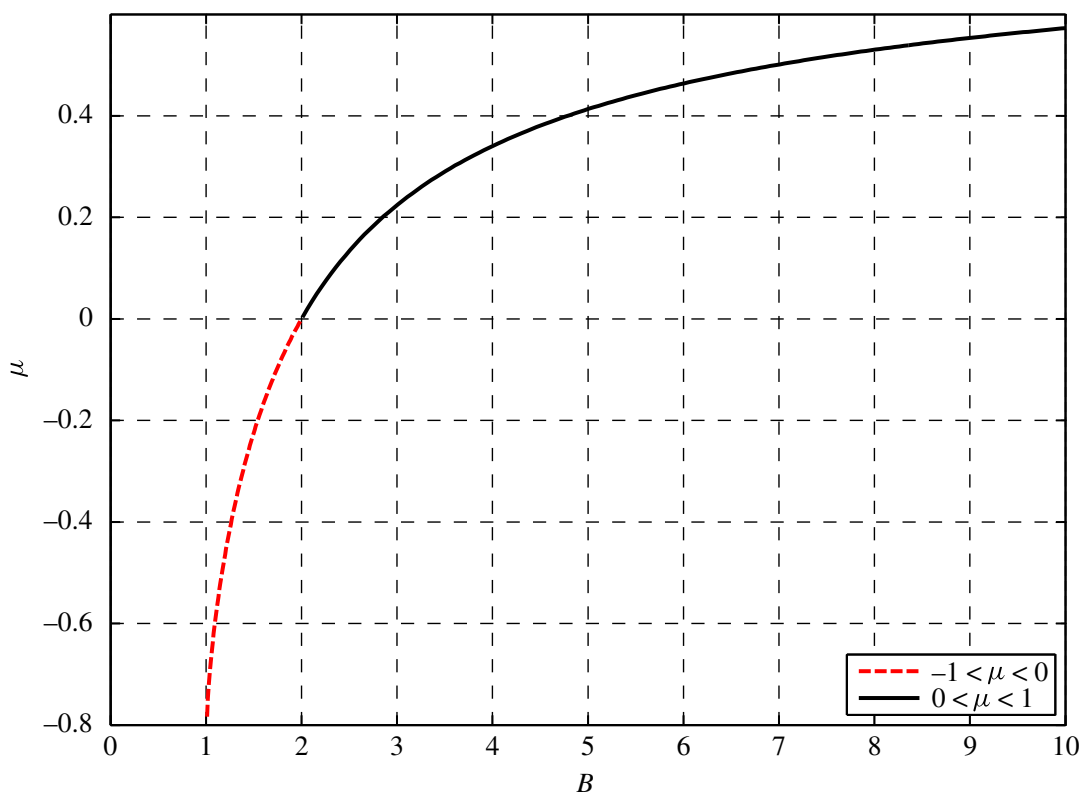

FIGURE 5. Relation between the parameters $\mu=(a-b) / \ell$ and $B=1 / k^{2}$ for the joining of two nanotubes.

that the bond length $\sigma$ of the carbon nanotubes is normalized to be $1 \AA$, we employ the tube radii values as calculated from the carbon nanotube polyhedral model as proposed by Cox and Hill [8], which are 2.2481, 2.5189 and $3.33634 \AA$ for the $(8,0),(9,0)$ and $(12,0)$ tubes, respectively. For prescribed $x_{0}$ and $x_{1}$, and assuming that the angle of inclination necessary to accommodate a single pentagon is $9.594^{\circ}$, the arc length $\ell$ is determined to be 4.9047 and $6.5300 \AA$, respectively. In the first region, where $B<2$ and $x_{0}>x_{1}$, we obtain $B=B_{1}=1.601$ for the connections of both the $(12,0)-$ $(8,0)$ and $(12,0)-(9,0)$ carbon nanotubes. Furthermore, for the second region, where $B>2$ and $x_{0}<x_{1}$, we obtain $B=B_{2}=2.650$ for both cases, and we note that from $k_{1}^{2}+k_{2}^{2}=1$ we deduce $B_{2}=B_{1} /\left(B_{1}-1\right)$. In the other words, there appears to be only one value of $B$ corresponding to the joining of any two carbon nanotubes in order to accommodate a single pentagonal ring. We obtain the same numerical values for $B_{1}$ and $B_{2}$ in consequence of the solution being invariant under constant stretching transformations, that is, under any length scaling.

4.2. A carbon nanotube and a flat graphene sheet For future nanoelectromechanical signalling devices, graphene sheets may be needed as the platform to transmit signals to other materials through joined carbon nanotubes. In this section we consider the problem of the perpendicular joining between a carbon nanotube and a flat graphene sheet. In particular, we use variational calculus to determine the curve adopted by the line connecting a horizontal plane and a vertical carbon nanotube such that the arc length of the curve and the size of the defect in the graphene sheet are 


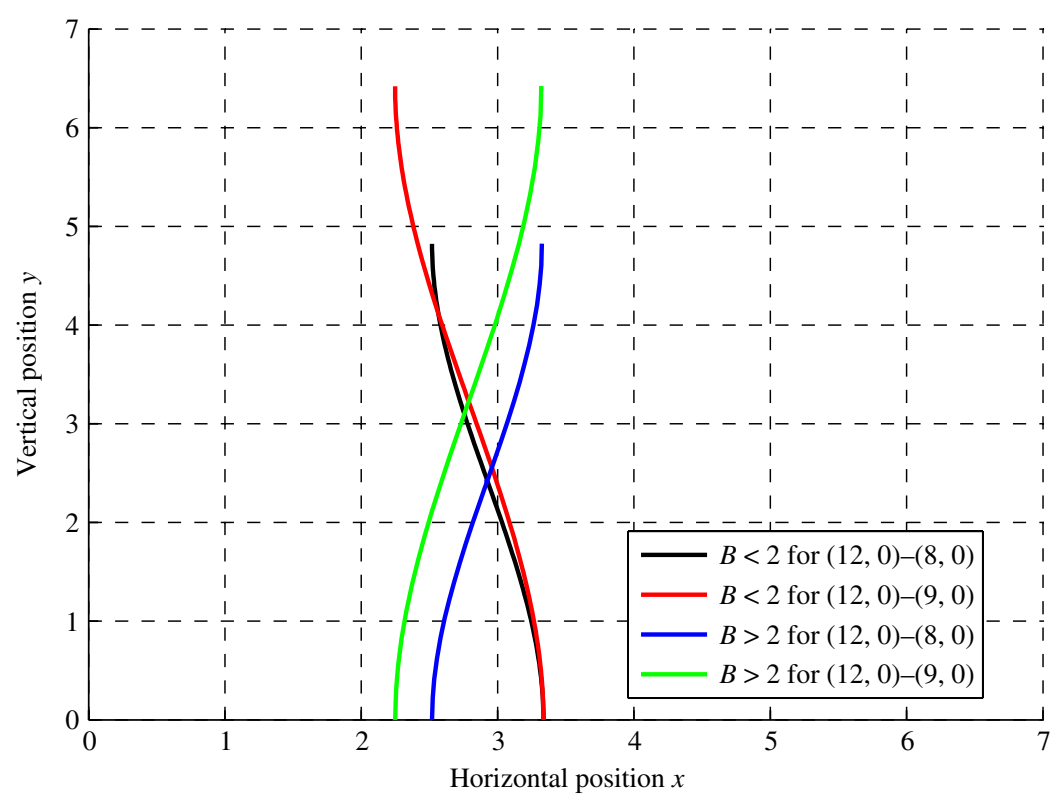

FIGURE 6. Graphs of joins $y=y(x)$ for two carbon nanotubes $(12,0)-(8,0)$ and $(12,0)-(9,0)$ where the angle between the two tube axes is assumed to be $9.594^{\circ}$.

specified. It is important to note that the distance of the carbon nanotube from the graphene sheet is not prescribed and is determined as part of the solution.

We position the graphene sheet in the $(x, z)$-plane, assuming a circular defect of radius $x_{0}$ centred on the origin. We also assume that a nanotube of radius $x_{1}$ is located with its axis colinear with the $y$-axis, starting from an unknown positive distance $y_{1}$ above the $(x, z)$-plane. Since the defect and the nanotube are assumed to be rotationally symmetric about the $y$-axis, we can consider this as a problem in the two-dimensional $(x, y)$-plane. The connecting covalent bonds are assumed to join the points on the graphene defect, $\left(x_{0}, 0\right)$, and the nanotube, $\left(x_{1}, y_{1}\right)$, and have a total prescribed arc length $\ell$. The angles at the boundaries of the join region are $\theta_{0}=0$ and $\theta_{1}=\pi / 2$. Two likely model configurations are illustrated in Figure 7.

Here, we observe that (3.7) coincides with (3.10) for the value $k=1 / \sqrt{2}$. We denote the value of $\mu$ at this point by $\mu_{0}$ and we have

$$
\mu_{0}=2\left(\frac{E(1 / \sqrt{2})}{K(1 / \sqrt{2})}\right)-1=0.4569465810 \ldots
$$

We begin by examining some general features of the solutions to Models I and II, and then subsequently we examine a particular carbon nanotube-graphene join. The different regions of the solution can be seen by examining (3.7) and (3.10), as shown in Figure 8, in which for convenience we plot $\mu$ against $B$. In this plot, three distinct regions are evident. First, there is the region $2 / \pi<\mu<1$, which corresponds to joins 


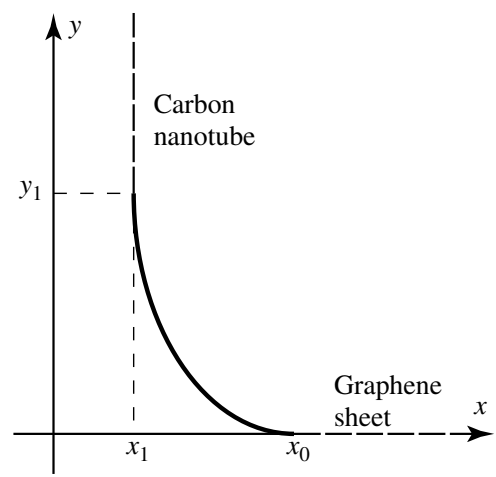

Model I

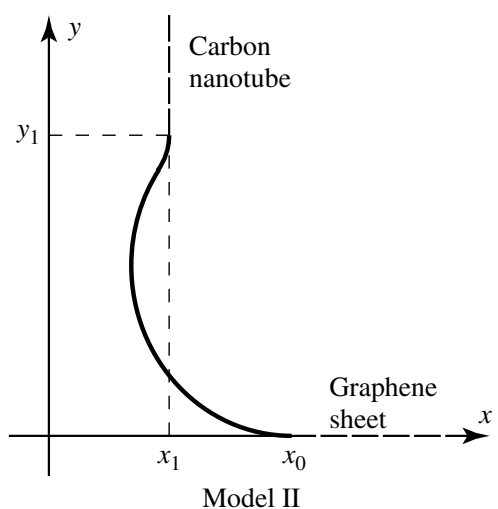

Model II

FIGURE 7. Geometries for Model I (join region has only positive curvature) and Model II (join region has both positive and negative curvatures) for the joining of a nanotube and graphene sheet.

with arc length $\ell$ less than a quarter of a circle circumference of radius $x_{0}-x_{1}$. In this case the constant $B$ is negative, which corresponds to a negative value of $\alpha$ and an imaginary modulus $k$ for the elliptic integrals. The solution asymptotes with the line $\mu=1$, and crosses the vertical axis at the point $\mu=2 / \pi$, which corresponds to the solution degenerating to constant curvature (that is, a circular join). The second region exists for $\mu_{0} \leq \mu<2 / \pi$, where $\mu_{0}$ is given by (4.1) and corresponds to Model I when the arc length $\ell$ of the join is greater than the quarter circumference of a circle of radius $x_{0}-a$. In this region, $0<B \leq 2$, and therefore $\alpha$ is always positive and the modulus $k$ is strictly real. The third and final region applies to the range $-1<\mu<\mu_{0}$, and corresponds to Model II. This model is invoked when the arc length $\ell$ is much greater than the quarter circumference of a circle of radius $x_{0}-a$, and therefore a change of curvature is necessary to accommodate the join. For Model II, the constant $B$ is restricted to the range $1<B \leq 2$, and therefore again $\alpha$ is strictly positive. As can be seen from Figure 8 , the constant $B$ never takes a value greater than 2 for any of the solution regions.

We assume a fixed arc length $\ell=1$ and a graphene attachment point $x_{0}=1$. We then allow the tube radius $a$ to take values between 0.1 and 0.9 , in increments of 0.1 . In this configuration, $\mu=1-a$ and the resulting joins are shown in Figure 9. As can be seen from this figure, for the three cases when $2 / \pi<\mu<1$, Model I is used with a negative value of $1 / k^{2}$. For the cases when $\mu_{0}<\mu<2 / \pi$, Model I is again used, however in these cases $0<1 / k^{2}<2$. Finally, in the cases when $-1<\mu<\mu_{0}$, Model II provides the solution.

4.3. A carbon nanotube and a $\mathbf{C}_{60}$ fullerene Similar in structure to graphene, $\mathbf{C}_{60}$ fullerenes are composed of linked hexagonal rings, which also contain pentagonal rings that are required in order to close the spherical surface. Nanobuds are believed to possess interesting properties, and they are envisaged to arise from the combination of a single-walled carbon nanotube and a $\mathrm{C}_{60}$ fullerene. Nanobuds were 


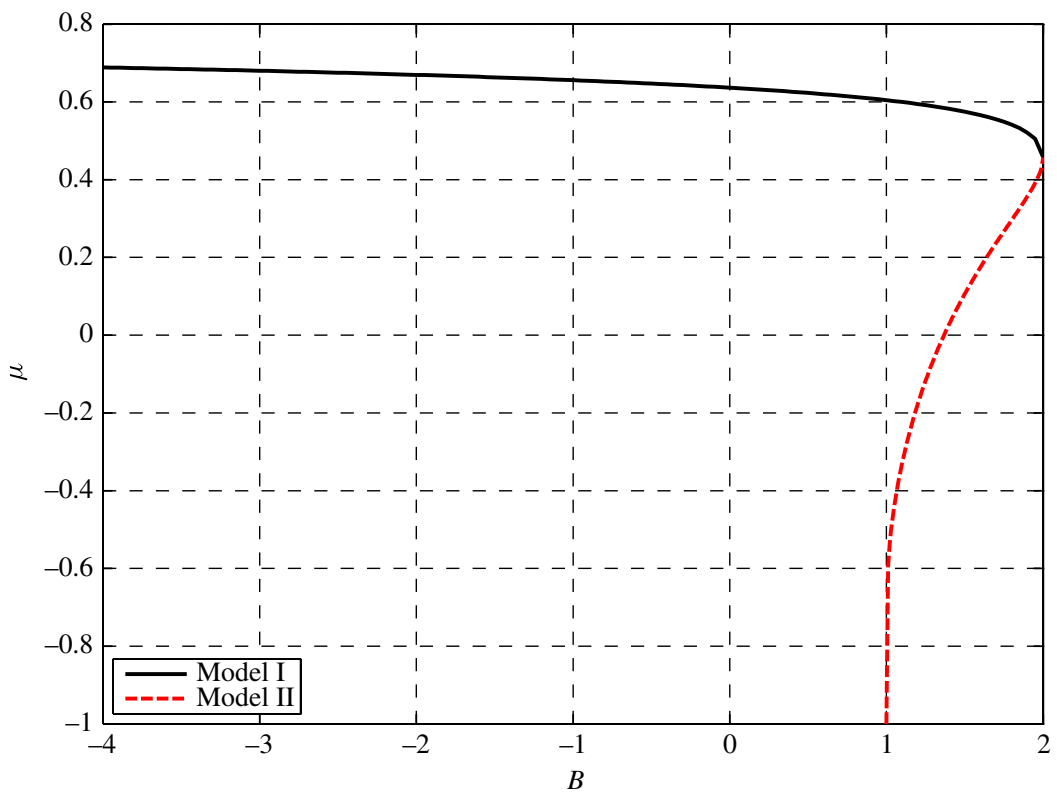

FIGURE 8. For the nanotube-graphene sheet join, the relation between the characteristic parameter $\mu=\left(x_{0}-a\right) / \ell$ and constant $B=1 / k^{2}$ for both Models I and II.

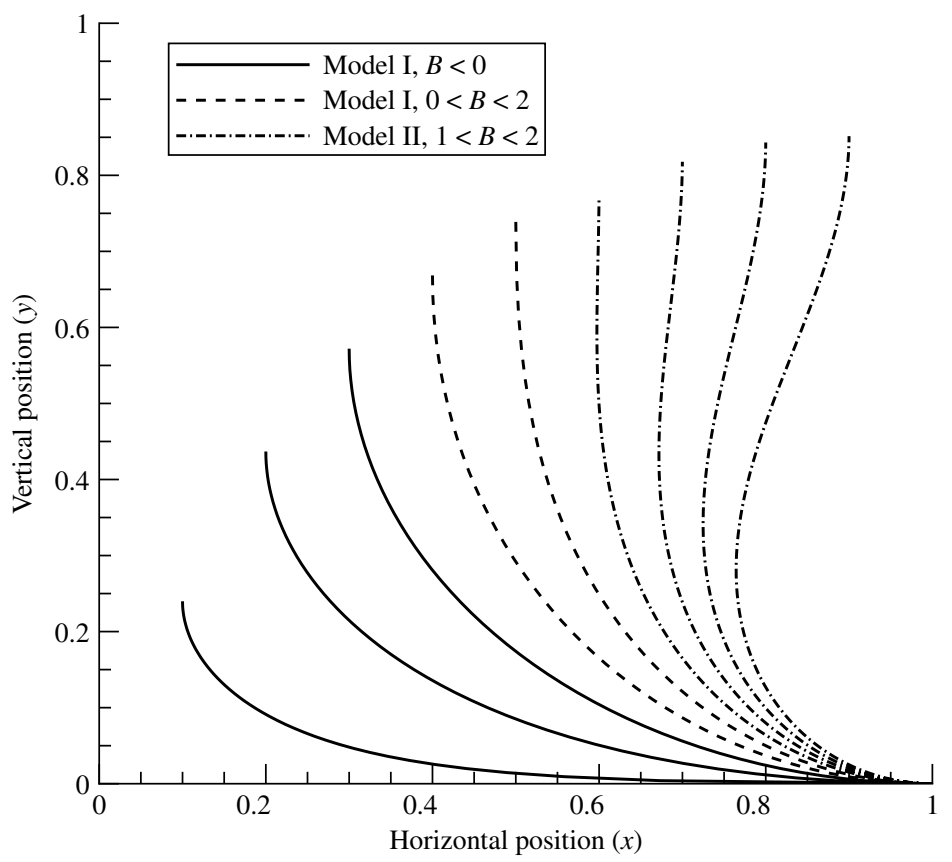

FIGURE 9. Nondimensional plots of joins $y=y(x)$ for various values of $\mu$ for a nanotube and a flat graphene sheet. 
first experimentally observed in a ferrocene-carbon monoxide system by Nasibulin et al. [15] from transmission electron microscopy. Furthermore, these authors also proposed a one-step continuous process for the synthesis of carbon nanotubes with covalently attached fullerenes. However, we note that a dynamical process involving the combination of these two carbon nanostructures was first accidentally observed by Zhao et al. [24]. They considered a fullerene encapsulated inside the nanotube, and a nanobud forming during this process. Nanobuds are believed to be promising fieldemission devices, since the off-plane of the fullerenes on the mat of carbon nanotubes can increase the surface area. In the continuous model, the calculus of variations is utilized to determine the junction for nanobuds. The defects on the fullerene and the carbon nanotube are assumed to be perfectly symmetrical, so that the problem can again be considered in the two-dimensional plane. Again, since the curvature can be both positive and negative, depending on the gap between these two nanostructures, two models are examined. We note that due to the three-dimensional structure and the pentagon-hexagon network of the fullerenes, the boundary conditions are slightly more complicated than those used for the flat hexagonal graphene as presented in Section 4.2.

Two distinct models are determined here and are illustrated in Figure 10. We position a $\mathrm{C}_{60}$ fullerene of radius $b$ in the $(x, z)$-plane centred at the origin. A carbon nanotube of radius $a$ is located with its axis colinear with the $y$-axis, starting from an unknown positive distance above the $(x, z)$-plane which we denote by $y_{1}$. Since the defect and the nanotube are rotationally symmetric about the $y$-axis, we can consider this as a problem in the two-dimensional $(x, y)$-plane. The total prescribed arc length $\ell$ is assumed to connect the defect at $(b \cos \psi, b \sin \psi)$ and the tube at $\left(a, y_{1}\right)$, where $\psi$ is the angle of attachment to the $\mathrm{C}_{60}$ fullerene as shown in Figure 10. The angles at the boundaries of the join region are $\theta_{0}$, which is varied, and $\theta_{1}=\pi / 2$.

In this case, we observe that equation (3.7) of Model I coincides with equation (3.10) of Model II for the values $k=1 / \sqrt{2}$ and $k=[(1-\sin \psi) / 2]^{1 / 2}$. When $k=1 / \sqrt{2}$, the value of $\mu$ is denoted by $\mu_{1}$ and given by

$$
\mu_{1}=2\left(\frac{E(1 / \sqrt{2})-E\left(\sin ^{-1}(\sqrt{1-\sin \psi}), 1 / \sqrt{2}\right)}{K(1 / \sqrt{2})-F\left(\sin ^{-1}(\sqrt{1-\sin \psi}), 1 / \sqrt{2}\right)}\right)-1
$$

When $k=[(1-\sin \psi) / 2]^{1 / 2}$, the value of $\mu$ is denoted by $\mu_{2}$ and given by

$$
\mu_{2}=2\left(\frac{E(\sqrt{(1-\sin \psi) / 2})-E\left(\sin ^{-1}(1 / \sqrt{1-\sin \psi}), \sqrt{(1-\sin \psi) / 2}\right)}{K(\sqrt{(1-\sin \psi) / 2})-F\left(\sin ^{-1}(1 / \sqrt{1-\sin \psi}), \sqrt{(1-\sin \psi) / 2}\right)}\right)-1
$$

Figure 11 shows the relation between the parameter $\mu=(b \cos \psi-a) / \ell$ and a parameter $B=1 / k^{2}$, and this graph can be divided into two main regions. The first region is $\mu<\mu_{0}$, which corresponds to $B<2$, and the second region is $\mu>\mu_{0}$, which corresponds to $B>2 /(1-\sin \psi)$, where $\mu_{0}$ is the asymptotic value for $\mu$ as $k$ tends to 


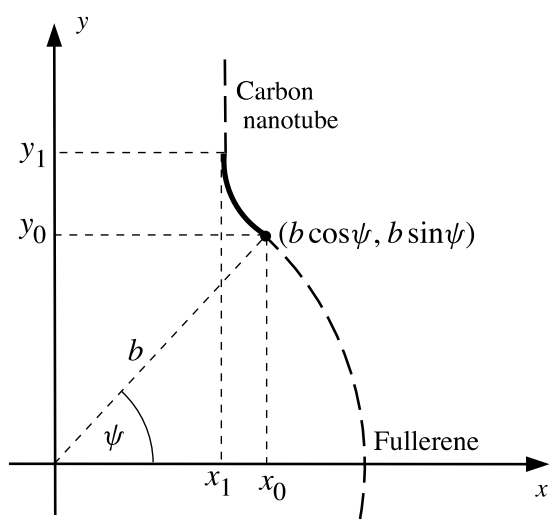

Model I

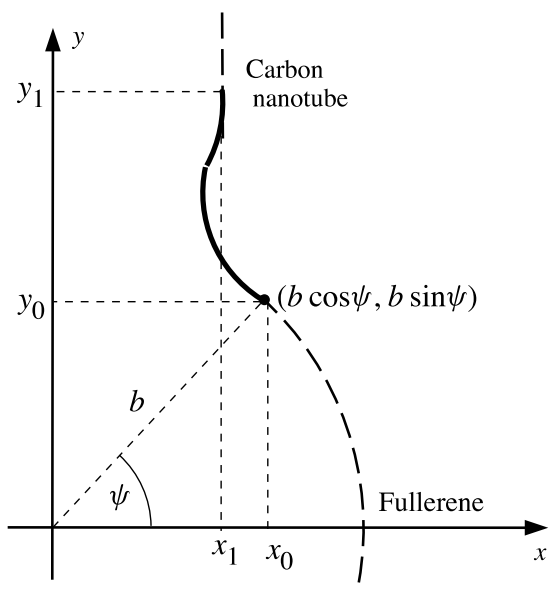

Model II

Figure 10. Geometries for Model I (join region has only positive curvature) and Model II (join region has both positive and negative curvatures) for the joining between a nanotube and fullerene.

zero, where it is shown to be

$$
\mu_{0}=1+\frac{\sqrt{2}(1-\sqrt{2} \cos \omega)}{\ln [(\sqrt{2}-1) / \tan (\omega / 2)]},
$$

where $\omega=\pi / 4-\psi / 2$. We note that these two values of $B$ arise when (3.7) and (3.10) coincide, as mentioned earlier.

The region $\mu<\mu_{0}$ can be divided into three subregions. The first subregion is $\mu_{3}<\mu<\mu_{0}$, where $\mu_{3}$ is the asymptotic value of $\mu$ as $k$ tends to infinity. We find that the solution asymptotes with the line $\mu=\mu_{0}$ and crosses the vertical axis at the point $\mu_{3}$, where $\mu_{3}$ can be analytically determined and is found to be

$$
\mu_{3}=\frac{1-\cos \psi}{\psi} \text {. }
$$

In this case, the parameter $B$ is negative, which corresponds to a negative value of $\alpha$ and an imaginary modulus $k$ for the elliptic functions. The second subregion is $\mu_{1}<\mu<\mu_{3}$, which corresponds to $0<B \leq 2$, so that $\alpha$ is always positive and the modulus $k$ is strictly real. The final subregion, which is obtained from Model II, applies for the range $-1<\mu<\mu_{1}$ which corresponds to $1<B \leq 2$, and again, $\alpha$ is always positive.

The region of $\mu>\mu_{0}$ can be divided into two subregions, which are $\mu_{0}<\mu<\mu_{2}$ from Model I and $\mu_{2}<\mu<1$ from Model II. The value of $B$ is always positive in both of these subregions, and it corresponds to a negative value of $\alpha$ and a complex value of angle $\phi$ of the form $\phi=-\pi / 2+i \varphi$. We note that in this region the curvature changes sign from negative to positive.

We also note that the values $\mu$ given in (3.7) and (3.10) are dependent on the initial angle $\psi$ of the $\mathrm{C}_{60}$ fullerene, as depicted in Figure 12. For the case $\psi=0$, a 


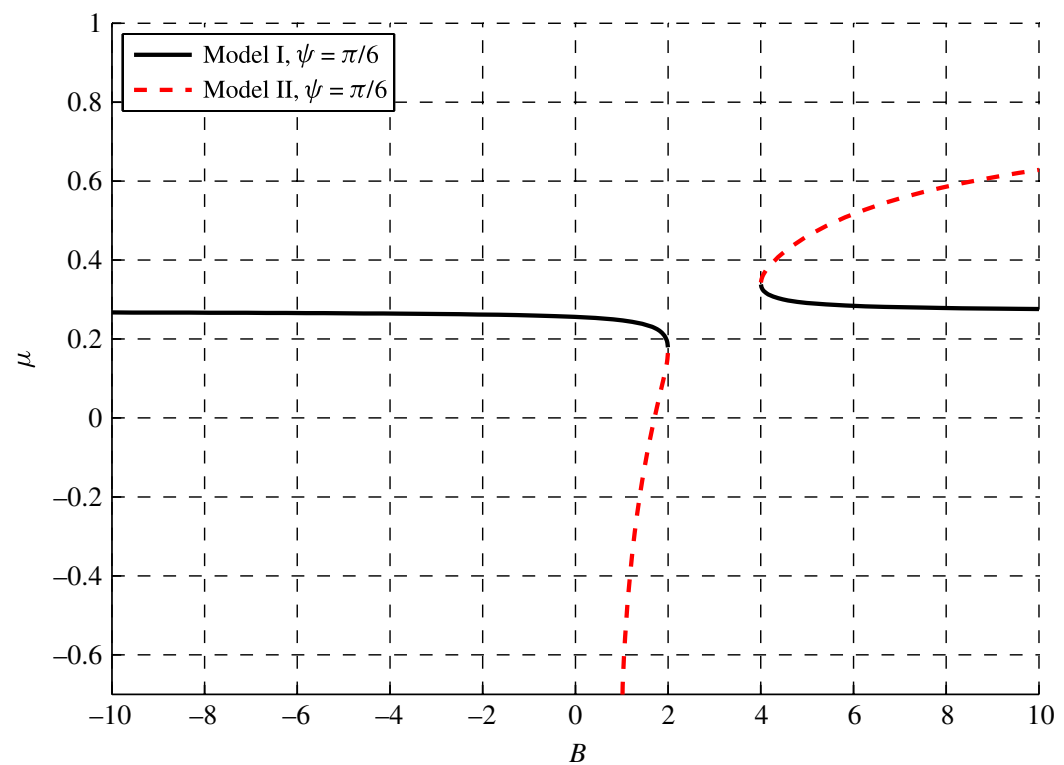

FIGURE 11. Relation between the parameters $\mu=(b \cos \psi-a) / \ell$ and $B=1 / k^{2}$ for both Model I and Model II.

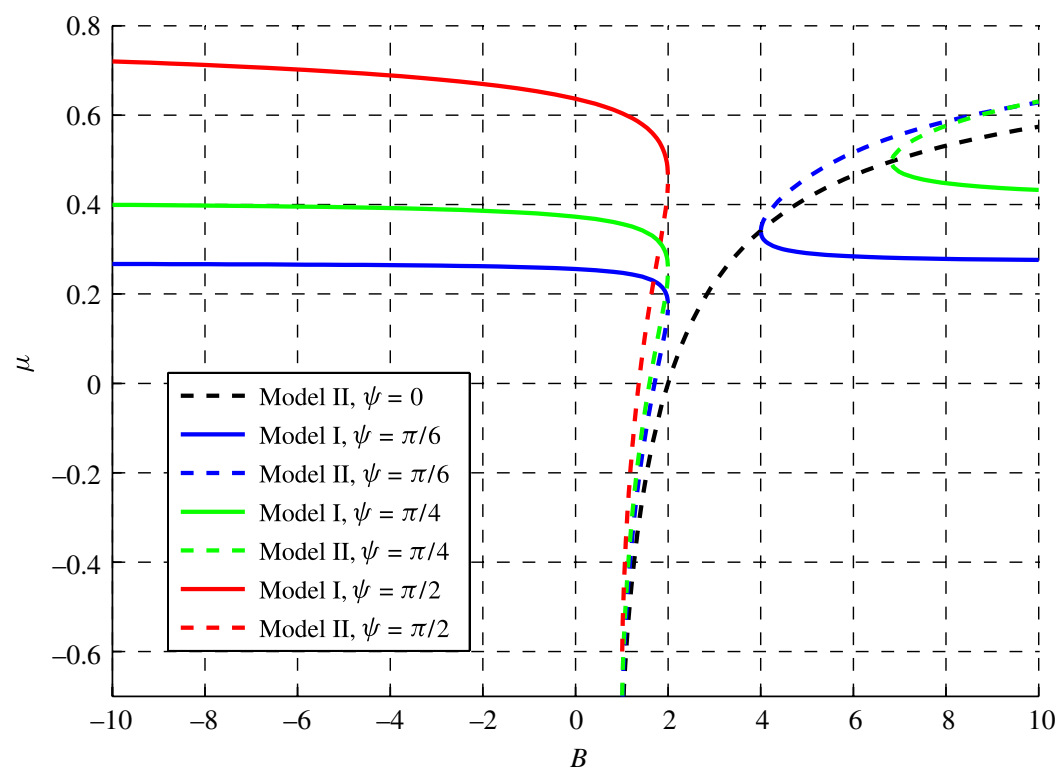

FIGURE 12. Relation between the parameters $\mu$ and $B$ when $\psi=0, \pi / 6, \pi / 4, \pi / 2$ for the joining between a nanotube and fullerene. (Colour available online.) 


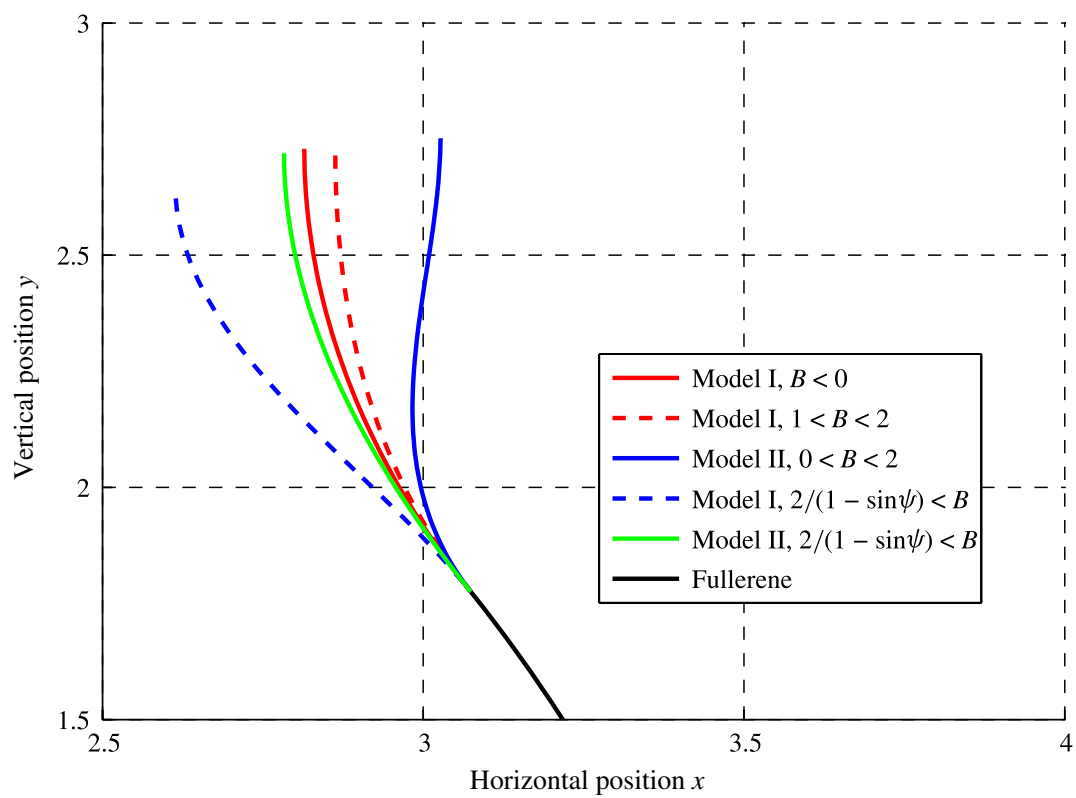

Figure 13. Plots of joins $y=y(x)$ for the values $B=-1,1.9,1.8,2.5$, 5 , which belong to the shown ranges, with $\psi$ assumed to be $\pi / 6$, for the joining of a nanotube and fullerene. (Colour available online.)

hemispherical fullerene, the gradients at the attachment points of the fullerene and the carbon nanotube are $\infty(-\infty)$. Therefore, the curvature of the arc has to change sign, and consequently, only Model II is applicable. This is shown in Figure 12, with the black dashed curve: $\mu_{0}$ becomes zero, and $\mu_{1}$ and $\mu_{2}$ meet at $B=2$. In the case $0<\psi<\pi / 2$, the solution for $\mu$ can occur in both of the two main regions of Model I and Model II as described previously. However, the second region, $\mu>\mu_{0}$, moves and goes to infinity as $\psi$ tends to $\pi / 2$, which is shown in Figure 12 , with blue and green solid and dashed curves. Finally, for the extreme case when $\psi=\pi / 2$, only the first region $\mu<\mu_{0}$ can be found, with $\mu_{0}=1$. The point of attachment at the $\mathrm{C}_{60}$ fullerene has a gradient of $\infty$ and the solution is similar to the case of joining a carbon nanotube to a flat graphene sheet as described in Section 4.2.

We now apply the solution of the continuous approach to a nondimensionalized situation which is shown in Figure 13. In this case, a fixed arc length is assumed to be $\ell=1$, the initial angle of the fullerene is assumed to be $\psi=\pi / 6$ and the values of $B$ are taken from the graph shown in Figure 12, by blue solid and dashed curves. We choose five values of $B$ for the five possible cases of Model I and Model II.

4.4. Carbon nanotubes and carbon nanocones Carbon nanostructures are believed to be unique materials which may be used as components to create many novel nanodevices. Most of the current research effort centres on the use of carbon nanotubes and $\mathrm{C}_{60}$ fullerenes. To date there has been very little work undertaken on carbon nanocones. One reason for this is that only a small number of carbon nanocones are 
TABLE 1. Relation between the number of pentagons $N_{p}$ and the open angle $\gamma$ (two decimal places) for carbon nanocones.

\begin{tabular}{cccccccc}
\hline$N_{p}$ & 0 & 1 & 2 & 3 & 4 & 5 & 6 \\
\hline$\gamma$ & $180^{\circ}$ & $112.89^{\circ}$ & $83.62^{\circ}$ & $60^{\circ}$ & $38.94^{\circ}$ & $19.19^{\circ}$ & $0^{\circ}$ \\
\hline
\end{tabular}

produced during synthesis [19]. Carbon nanocones were first discovered by Ge and Sattler [13] and subsequently synthesised by Krishnan et al. [14]. They are formed from a graphene sheet with the disclination number of pentagons $N_{p}=1,2,3,4$ or 5 , so that there are five possible nontrivial ways to create a carbon nanocone. As a consequence of Euler's theorem (faces plus vertices equals edges plus 2), the cap of a carbon nanotube consists of six pentagons, and we note that for $N_{p}=6$ we obtain a capped carbon nanotube, and $N_{p}=0$ corresponds to a graphene sheet. For a regular polyhedron comprising only hexagons and pentagons, Euler's theorem immediately gives $N_{p}=6$ for any closed cap, so that, for example, any fullerene $\mathrm{C}_{m}$ contains precisely 12 pentagons. Assuming that the cone vertex angle is given by $\gamma$, the two trivial and five nontrivial values of $\gamma$ are shown in Table 1.

Most research on carbon nanocones deals with their electronic structure [7, 16], and it is generally believed that the different number of pentagons in carbon nanocones is the key to the puzzle of nucleation in atomic construction. Pincak and Osipov [16] found that the electron states depend on the position of the pentagons. Moreover, because of their local density properties, Charlier and Rignanese [7] have proposed carbon nanocones as the ideal candidates for the probes of scanning tunnelling microscopes.

Again, two distinct models are determined here and illustrated in Figure 14. We position the nanocone of base radius $r$ such that the cone vertex is assumed to be located at the origin of the $(x, z)$-plane as indicated in Figure 14. A carbon nanotube of radius $a$ is located with its axis colinear with the $y$-axis starting from an unknown positive distance above the $(x, z)$-plane, which we denote by $y_{1}$. Since the nanocone and the nanotube are rotationally symmetric about the $y$-axis, we can consider this as a problem in the two-dimensional $(x, y)$-plane. The total prescribed arc length $\ell$ is assumed to connect a defect at $(r, h)=(b \sin (\gamma / 2), b \cos (\gamma / 2))$ and the tube at $\left(a, y_{1}\right)$, where $\gamma$ is the cone angle and $b=r \csc (\gamma / 2)$ as shown in Figure 14. We note that $(b \sin (\gamma / 2), b \cos (\gamma / 2))$ and $\left(a, y_{1}\right)$ are the nearest atomic positions of the joined atoms on the cone base and on the tube open end, respectively, and there are five possibilities for the cone angle $\gamma$. In this case, we have $\theta_{0}=-\gamma / 2$ and $\theta_{1}=\pi / 2$.

Here, equation (3.7) of Model I coincides with equation (3.10) of Model II for the values $k=1 / \sqrt{2}$ and $k=\{[1-\sin (\gamma / 2)] / 2\}^{1 / 2}$. When $k=1 / \sqrt{2}$, the value of $\mu$ is denoted by $\mu_{1}$ and it can be shown to be given by

$$
\mu_{1}=2\left(\frac{E(1 / \sqrt{2})-E\left(\sin ^{-1}(\sqrt{1-\sin (\gamma / 2)}), 1 / \sqrt{2}\right)}{K(1 / \sqrt{2})-F\left(\sin ^{-1}(\sqrt{1-\sin (\gamma / 2)}), 1 / \sqrt{2}\right)}\right)-1 .
$$




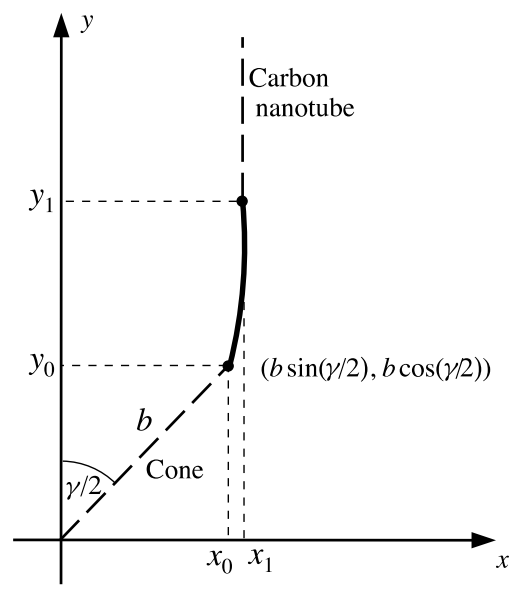

Model I

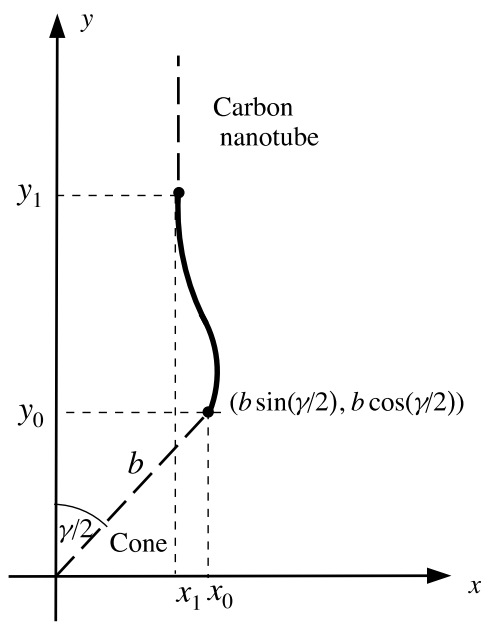

Model II

FIGURE 14. Geometries for Model I (join contains only positive curvature) and Model II (join has both positive and negative curvatures) for the joining between a nanotube and nanocone.

When $k=\{[1-\sin (\gamma / 2)] / 2\}^{1 / 2}$, the value of $\mu$ is denoted by $\mu_{2}$ and given by

$$
\mu_{2}=2\left(\frac{E(\sqrt{[1-\sin (\gamma / 2)] / 2})-E\left(\sin ^{-1}[1 / \sqrt{1-\sin (\gamma / 2)}], \sqrt{[1-\sin (\gamma / 2)] / 2}\right)}{K(\sqrt{[1-\sin (\gamma / 2)] / 2})-F\left(\sin ^{-1}[1 / \sqrt{1-\sin (\gamma / 2)}], \sqrt{[1-\sin (\gamma / 2)] / 2}\right)}\right)-1
$$

Firstly, the solution, which is characterized by the nondimensional parameter $\mu=[a-b \sin (\gamma / 2)] / \ell$ subject to the constraint $-1<\mu<1$, is examined. In Figure 15, we show the relation between the parameter $\mu$ and a parameter $B=1 / k^{2}$, and this graph can be divided into two main regions. The first region is $\mu<\mu_{0}$, which corresponds to $B<2$, and the second region is $\mu>\mu_{0}$, which corresponds to $B>2 /[1-\sin (\gamma / 2)]$, where $\mu_{0}$ is the asymptotic value for $\mu$ as $k$ tends to zero, which is

$$
\mu_{0}=1+\frac{\sqrt{2}(1-\sqrt{2} \cos \omega)}{\ln [(\sqrt{2}-1) / \tan (\omega / 2)]}, \quad \text { where } \omega=(\pi-\gamma) / 4
$$

The region $\mu<\mu_{0}$ can be divided into three subregions. The first subregion is $\mu_{3}<\mu<\mu_{0}$, where again $\mu_{3}$ is the asymptotic value of $\mu$ as $k$ tends to infinity, which is found to be

$$
\mu_{3}=\frac{2}{\gamma}\left(1-\cos \frac{\gamma}{2}\right)
$$

In this case, the parameter $B$ is negative, which corresponds to a negative value of $\alpha$ and an imaginary modulus $k$ for the elliptic functions. The second subregion is $\mu_{1}<\mu<\mu_{3}$, which corresponds to $0<B \leq 2$, so that $\alpha$ is always positive and the modulus $k$ is strictly real. The final subregion, which is obtained from Model II, applies for the range $-1<\mu<\mu_{1}$, which corresponds to $1<B \leq 2$, and again $\alpha$ is always positive. 


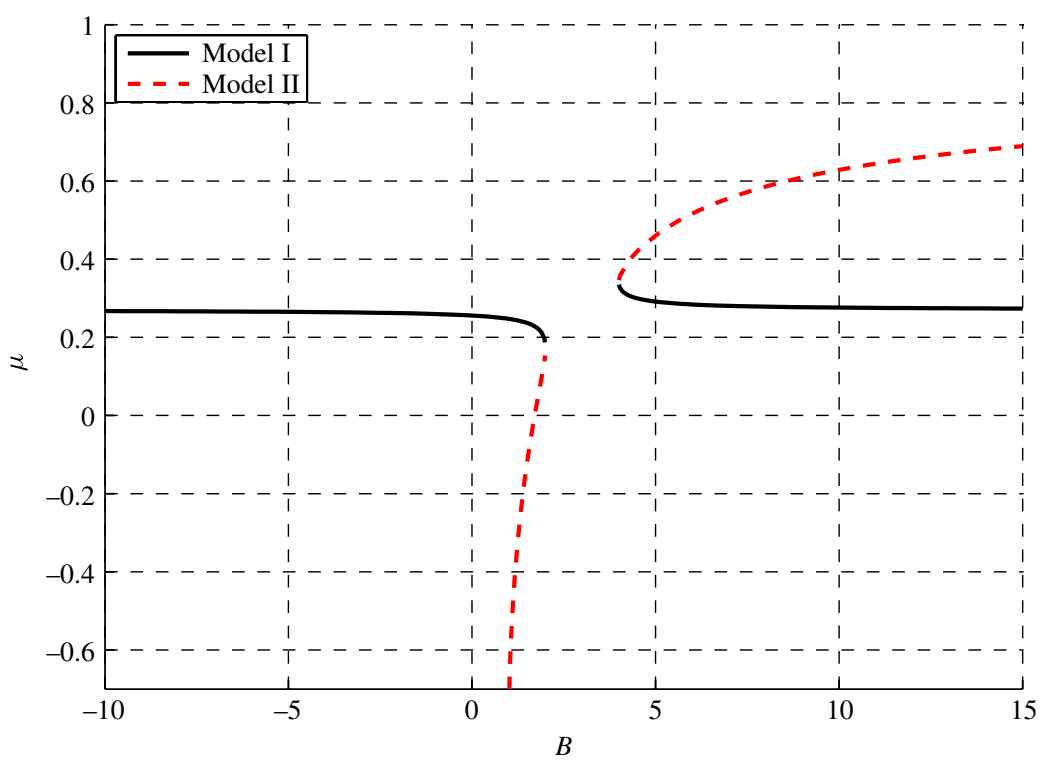

FIGURE 15. For the nanotube-nanocone join, the relation between the parameters $\mu=[a-b \sin (\gamma / 2)] / \ell$ and $B=1 / k^{2}$ as obtained for both Model I and Model II.

The region $\mu>\mu_{0}$ can be divided into two subregions, which are $\mu_{0}<\mu<\mu_{2}$ from Model I and $\mu_{2}<\mu<1$ from Model II. The value of $B$ is always positive in both of these subregions, and it corresponds to a negative value of $\alpha$ and a complex value of the angle $\phi$ of the form $\phi=\pi / 2+i \varphi$. We note that in this region the curvature changes sign from negative to positive. Furthermore, we note that the values of $\mu$ given in (3.7) and (3.10) also depend on the cone angle $\gamma$ and the region for $\mu>\mu_{0}$ moves away from the origin as $\gamma$ increases.

We now apply the solution of the continuous approach to a nondimensionalized situation which is shown in Figures 16(a) and 16(b) for Model I and Model II, respectively. For purposes of comparison, the cone heights are assumed to be equal and the cone radii are obtained from $r=h \tan (\gamma / 2)$. In addition, the fixed arc length is assumed to be $\ell=2$ for all five possible carbon nanocones. We note that a closer examination of the join regions shown in Figure 16 shows that they connect perfectly smoothly, as seen in Figure 17.

4.5. Two fullerenes of differing radii Two joined fullerenes, which are connected to form novel peanut-like nanostructures, are hollow structures which have the potential to encapsulate other molecules inside [22]. The first reported coalescence of two fullerenes by Ueno et al. [20], who employed a Stone-Wales calculation, found that any two $\mathrm{C}_{60}$ fullerenes can rearrange themselves to form a $\mathrm{C}_{120}$ molecule. Furthermore, Xia et al. [21] used molecular dynamics simulation to investigate the processes of dimerization and fusion reactions induced by two $\mathrm{C}_{60}$ molecules. They 


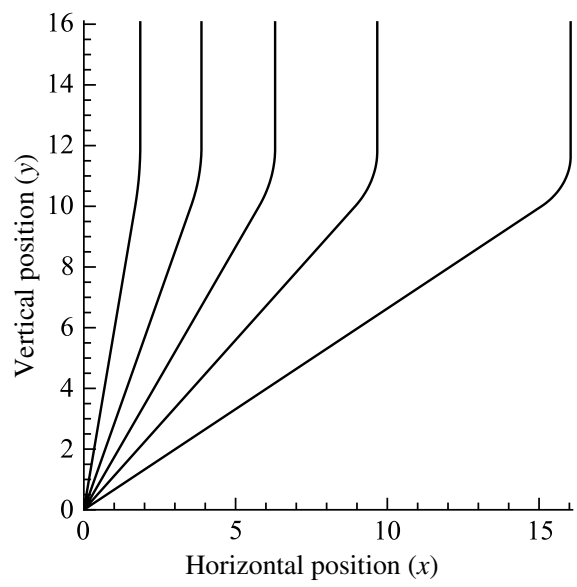

(a)

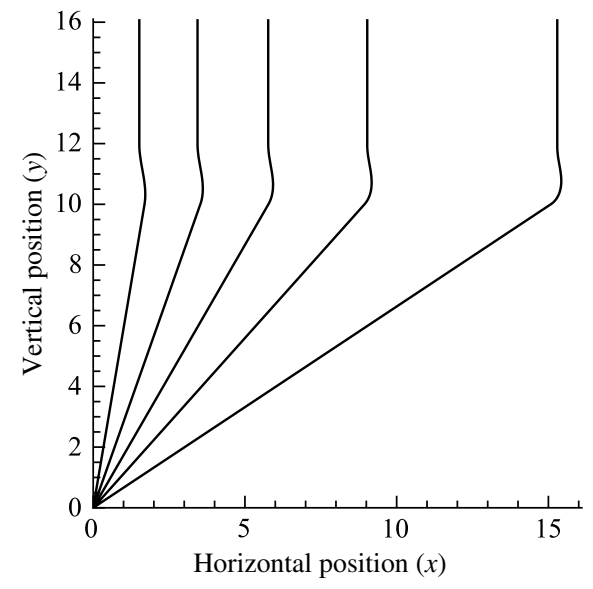

(b)

FIGURE 16. Plots of nanotube-nanocone joins $y=y(x)$ for five possible carbon nanocones with $\ell=2$ : (a) Model I where $B=-4.0$, and (b) Model II where $B=1.7$.

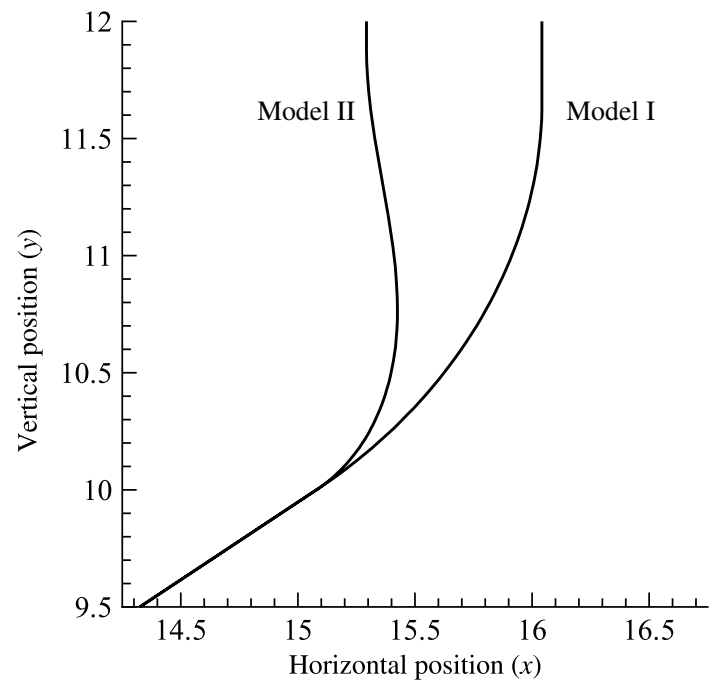

FIGURE 17. Magnified plot of nanotube-nanocone connection for $\gamma=112.9^{\circ}$ for both Models I and II. 
found that a dumbbell shaped molecule can be formed at low collision energies, and that when the energy is high enough a large $\mathrm{C}_{120}$ can be obtained. Subsequently, Yakobson and his collaborators [24-26] utilized a Stone-Wales method to determine the topology of coalescent fullerenes. They commented that finding the join between two buckyballs is a challenging task since the computational calculation can hardly go beyond a few hundred iterations. Therefore, in order to solve this challenging problem, an applied mathematical approach might be needed. One potential application of these combined structures is for the storage of hydrogen. Recently, using computer simulation, Ding et al. [10] proposed a design for foam-like structures which is based on joining single-walled carbon nanotubes to form three-dimensional architectures. These authors suggest that these novel structures might constitute an excellent hydrogen storage medium. Accordingly, in order to reduce the computational effort, the present calculus of variations approach might provide a more efficient means to model such storage devices.

Two distinct models are determined, illustrated in Figure 18. To represent the first fullerene, we position a circle of radius $a$ centred at the origin. The other fullerene is represented by a circle of radius $b$ located on the $y$-axis at a positive distance from the origin. The defects are assumed to be symmetric about the $y$-axis, such that the first fullerene terminates at an angle $\psi_{1}$ anticlockwise from the $x$-axis and the second fullerene terminates at an angle $\psi_{2}$ anticlockwise from the $x$-axis. The total prescribed arc length $\ell$ is assumed to connect the defects at the points $\left(a \cos \psi_{1}, a \sin \psi_{1}\right)$ and ( $b \cos \psi_{2}, L+b \sin \psi_{2}$ ). In this case, the angles at the boundaries of the join region are $\theta_{0}=-\psi_{1}$ and $\theta_{1}=-\psi_{2}$.

We also note that equation (3.7) of Model I coincides with equation (3.10) of Model II for the values $k=\left[\left(1-\sin \psi_{1}\right) / 2\right]^{1 / 2}$ and $k=\left[\left(1-\sin \psi_{2}\right) / 2\right]^{1 / 2}$. When $k=\left[\left(1-\sin \psi_{1}\right) / 2\right]^{1 / 2}$, we denote $k=k_{1}$ and $\mu=\mu_{1}$, which is given by

$$
\mu_{1}=2\left(\frac{E\left(k_{1}\right)-E\left(\phi_{3}, k_{1}\right)}{K\left(k_{1}\right)-F\left(\phi_{3}, k_{1}\right)}\right)-1,
$$

where $\phi_{3}=\sin ^{-1}\left(\sqrt{\left(1-\sin \psi_{2}\right) /\left(1-\sin \psi_{1}\right)}\right)$. When $k=\left[\left(1-\sin \psi_{2}\right) / 2\right]^{1 / 2}$, we denote $k=k_{2}$ and $\mu=\mu_{2}$, which is given by

$$
\mu_{2}=2\left(\frac{E\left(k_{2}\right)-E\left(\phi_{4}, k_{2}\right)}{K\left(k_{2}\right)-F\left(\phi_{4}, k_{2}\right)}\right)-1,
$$

where $\phi_{4}=\sin ^{-1}\left(\sqrt{\left(1-\sin \psi_{1}\right) /\left(1-\sin \psi_{2}\right)}\right)$. We note that $\sin \phi_{3}=\csc \phi_{4}$.

The solution, which is characterized by the nondimensional parameter $\mu=$ $\left(a \cos \psi_{1}-b \cos \psi_{2}\right) / \ell$, subject to the constraint $-1<\mu<1$, is illustrated in Figure 19 . Figure 19, black solid and dashed curves, can be divided into two main regions. The first region is $\mu<\mu_{0}$, which corresponds to $B<2 /\left(1-\sin \psi_{2}\right)$, and the second region is $\mu>\mu_{0}$, which corresponds to $B>2 /\left(1-\sin \psi_{1}\right)$. The parameter $\mu_{0}$ is obtained as

$$
\mu_{0}=1+2\left\{\frac{\cos \omega_{2}-\cos \omega_{1}}{\ln \left[\tan \left(\omega_{2} / 2\right) / \tan \left(\omega_{1} / 2\right)\right]}\right\}
$$

where $\omega_{1}=\pi / 4-\psi_{1} / 2$ and $\omega_{2}=\pi / 4-\psi_{2} / 2$. 


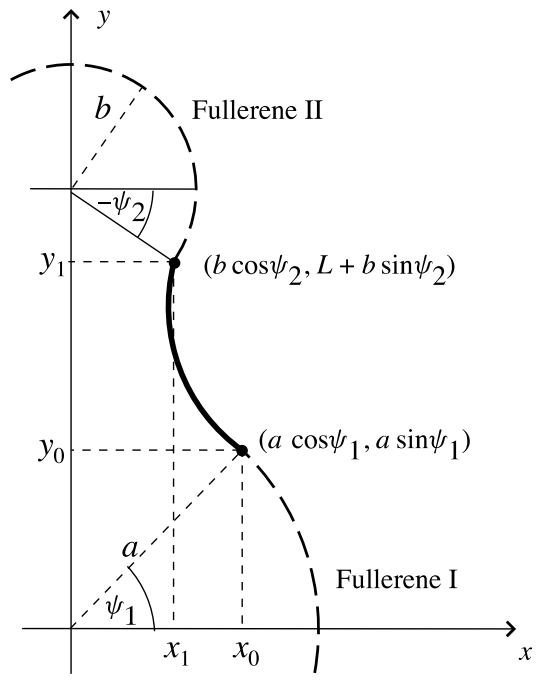

Model I

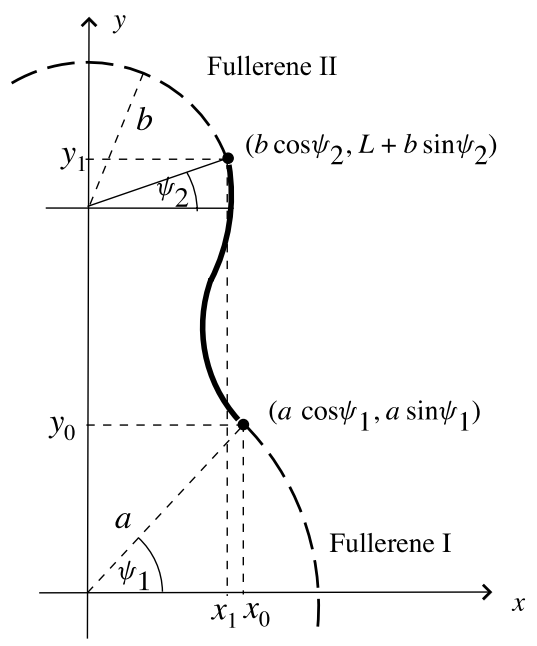

Model II

FIgure 18. Geometries for Model I, for which the join has only positive curvature, and Model II, which has both positive and negative curvature in the join region.

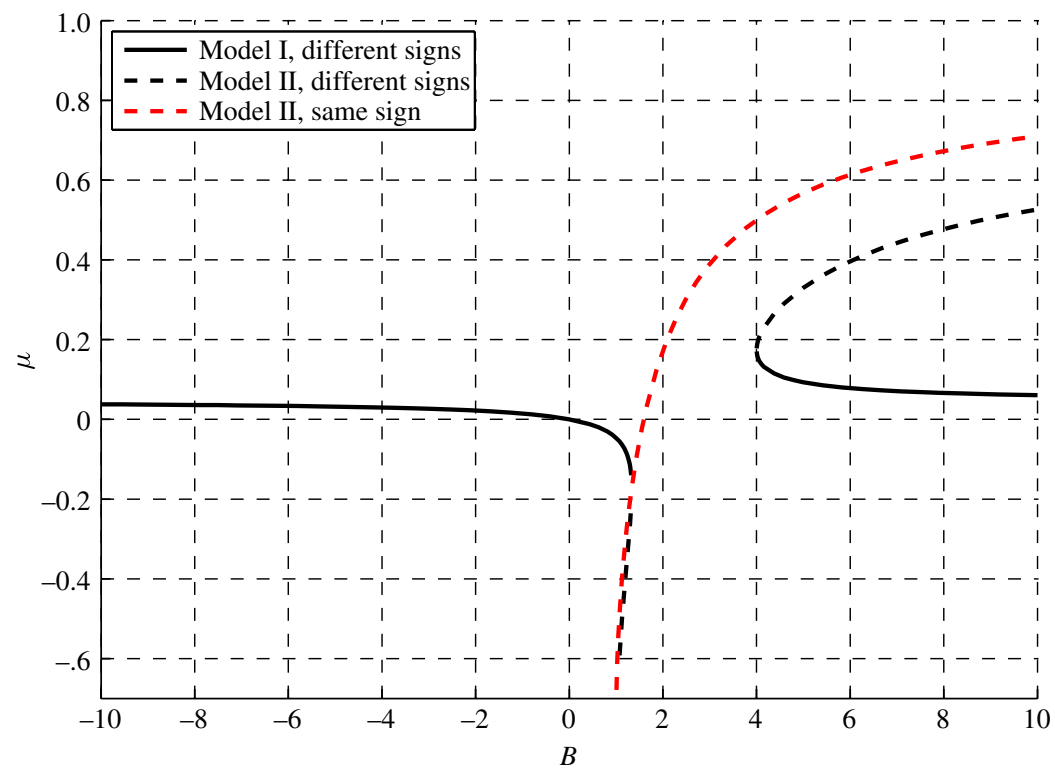

FIgURE 19. Relation between the parameters $\mu$ and $B$ for joining fullerenes when $\psi_{1}$ and $\psi_{2}$ have different signs, or have the same sign. (Colour available online.) 
The region where $\mu<\mu_{0}$ can be further divided into three subregions. The first of these is $\mu_{3}<\mu<\mu_{0}$, where $\mu_{3}$ is found to be

$$
\mu_{3}=\frac{\cos \psi_{2}-\cos \psi_{1}}{\psi_{1}-\psi_{2}}
$$

In the first subregion, the parameter $B$ is negative, which corresponds to a negative value of $\alpha$ and an imaginary modulus $k$ for the elliptic functions. The second subregion is identified for $\mu_{1}<\mu<\mu_{3}$, which corresponds to $0<B \leq 2 /\left(1-\sin \psi_{2}\right)$. In this subregion, $\alpha$ is always positive and the modulus $k$ is strictly real. The final subregion, which applies for Model II, is $-1<\mu<\mu_{1}$, which corresponds to $1<B \leq 2 /\left(1-\sin \psi_{2}\right)$, and again, $\alpha$ is always positive and $k$ is strictly real.

The region $\mu>\mu_{0}$ can be further divided into two subregions, which are $\mu_{0}<\mu<\mu_{2}$ for Model I and $\mu_{2}<\mu<1$ for Model II. The value of $B$ is always positive in both of these subregions, and it corresponds to a negative value of $\alpha$ and a complex value of angle $\phi$ of the form $\phi=-\pi / 2+i \varphi$. We note that in this region the curvature changes sign from negative to positive.

The values of $\mu$ given in equations (3.7) and (3.10) are also dependent on the initial angles $\psi_{1}$ and $\psi_{2}$ of the two fullerenes, where we assume $\psi_{1}, \psi_{2} \in(-\pi / 2, \pi / 2)$. When these two initial angles have the same sign (either both positive or both negative), the gradients at the attachment points of the two fullerenes are positive (negative). Therefore, the curvature of the arc has to change sign, and consequently, only Model II is applicable. This is shown in Figure 19, by the red dashed curve: $\mu_{0}$ becomes zero, and $\mu_{1}$ and $\mu_{2}$ meet at $B=2 /\left(1-\sin \psi_{2}\right)$. Generally, the solution for $\mu$ may exist in either of the two main regions of Model I and Model II, as described previously. For the case when $\psi_{1}=0$ and $\psi_{2}=\pi / 2$, only the first region $\mu<\mu_{0}$ can be found with $\mu_{0}=1$. The points of attachment of the two fullerenes have gradients of $\infty$ and the solution is similar to the case of joining a carbon nanotube to a flat graphene sheet as described in Section 4.2. On assuming $\psi_{2}=\pi / 2$ and varying the value of $\psi_{1}$, the point of attachment of the second fullerene above the origin has a gradient of $\infty$ and the solution is dependent on $\psi_{1}$, which is similar to the case of joining a carbon nanotube to a $\mathrm{C}_{60}$ fullerene, forming a nanobud, as described in Section 4.3.

For prescribed $a, b, \psi_{1}, \psi_{2}$ and $\ell$, we can numerically determine values of $B=1 / k^{2}$ and $\beta$. However, it is not a simple task to choose $a, b, \psi_{1}$ and $\psi_{2}$ and vary $\ell$ to produce values of $B$ which fall in all five regions of the two models, as described previously. For convenience, we choose five different values of $B$ corresponding to the five regions as shown in Figure 19, from which we obtain values of $\beta$. As shown in Figure 20, we assume that $a=5, \psi_{1}=\pi / 3, \psi_{2}=-\pi / 6$ and $\ell=3$; consequently, $b$ can be determined. In Figure 21, the final joining structures of two fullerenes from the two models are illustrated, where $a=5, \psi_{1}=\pi / 3, \psi_{2}=-\pi / 6$ and $\ell=3$.

\section{Conclusion}

The aim of this paper was to review the work of the present authors using conventional applied mathematical modelling to determine a continuous approximate 


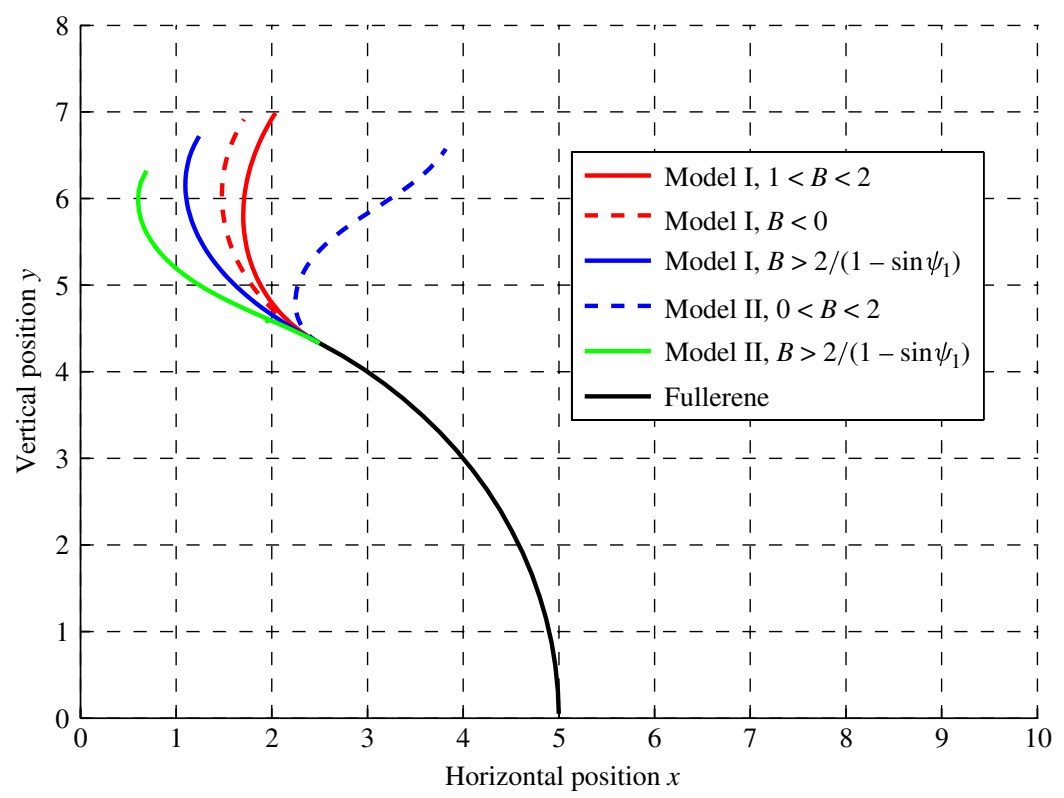

FIGURE 20. Plots of fullerene joins $y=y(x)$ for $B=-1,1.1$ and 20 for Model $\mathrm{I}$ and $B=1$ and 20 for Model II, corresponding to five regions, where $a=5, \psi_{1}=\pi / 3, \psi_{2}=-\pi / 6$ and $\ell=3$. (Colour available online.)

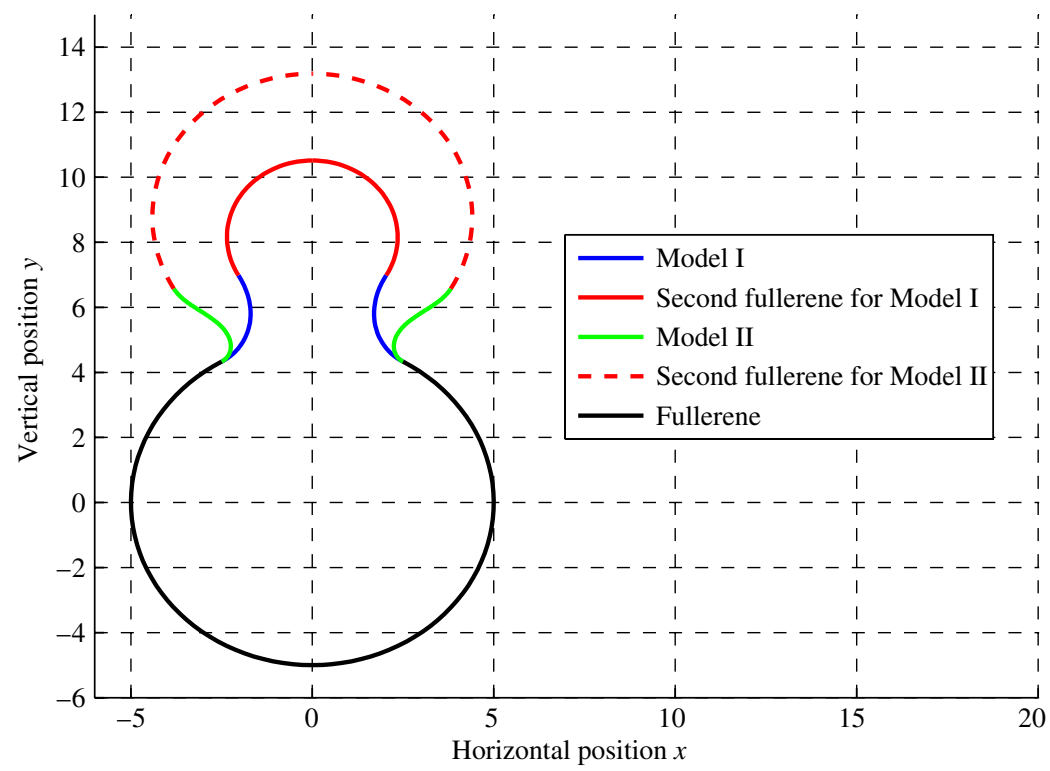

FIGURE 21. Illustrations for connections between two fullerenes by Model I and Model II, where $a=5$, $\psi_{1}=\pi / 3, \psi_{2}=-\pi / 6$ and $\ell=3$. (Colour available online.) 
analytical solution to the essentially discrete problem of the determination of the surface that joins two carbon nanostructures. Other than the isolated problem of joining two carbon nanotubes of distinct radii using a discrete geometrical approach [18, pp. 115-135], there are no general methods for such problems. The variational technique described here is an entirely novel procedure, based upon the notions that carbon nanostructures are virtually perfectly elastic and that their elastic energy can be represented by the surface curvature squared. In particular, we consider the joining problems involving: two carbon nanotubes of differing radii; a carbon nanotube and a flat graphene sheet; a carbon nanotube and a $\mathrm{C}_{60}$ fullerene; a carbon nanotube and a carbon nanocone; and two fullerenes of differing radii. We utilize a continuous model based on the calculus of variations, and we assume that each system is axially symmetric, so that the problem may be reduced to two dimensions. In reality, however, we recognise that at the atomic level the problems are fully three dimensional and are neither continuous nor axially symmetric. We render the approximate solutions presented here as describing the major dominant features of the problems, so that the real atomic structures can be described in terms of the deviations from the ideal models presented here. These continuous approaches require far less computational effort, but both computer simulations and experiments have yet to confirm the precise theoretical structures proposed here. However, these simple models based on certain assumptions appear to give rise to physically sensible approximations to highly complex structures and therefore the results are noteworthy.

\section{Acknowledgements}

D. Baowan gratefully thanks the Faculty of Science, Mahidol University. B. J. Cox and J. M. Hill acknowledge the support of the Australian Research Council through the Discovery Projects scheme and for providing an Australian Postdoctoral Fellowship for B. J. Cox.

\section{References}

[1] D. Baowan, B. J. Cox and J. M. Hill, "Two least squares analyses of bond lengths and bond angles for the joining of carbon nanotubes to graphenes", Carbon 45 (2007) 2972-2980; doi:10.1016/j.carbon.2007.09.045.

[2] D. Baowan, B. J. Cox and J. M. Hill, "A continuous model for the joining of two fullerenes", Philos. Mag. 88 (2008) 2953-2964; doi:10.1080/14786430802446682.

[3] D. Baowan, B. J. Cox and J. M. Hill, "Discrete and continuous approximations for nanobuds", Fuller. Nanotubes Carbon Nanostruct. 18 (2010) 160-177; doi:10.1080/15363830903586625.

[4] D. Baowan, B. J. Cox and J. M. Hill, "Modelling the joining of nanocones and nanotubes", J. Math. Chem. 49 (2011) 475-488; doi:10.1007/s10910-010-9753-8.

[5] D. Baowan, B. J. Cox and J. M. Hill, "Modeling the join curve between two co-axial carbon nanotubes", J. Appl. Math. Phys. (ZAMP) 63 (2011) 331-338; doi:10.1007/s00033-011-0140-5.

[6] P. F. Byrd and M. D. Friedman, Handbook of elliptic integrals for engineers and scientists, 2nd edn (Springer, Berlin, 1971).

[7] J.-C. Charlier and G.-M. Rignanese, "Electronic structure of carbon nanocones", Phys. Rev. Lett. 86 (2001) 5970-5973; doi:10.1103/PhysRevLett.86.5970. 
[8] B. J. Cox and J. M. Hill, "Exact and approximate geometric parameters for carbon nanotubes incorporating curvature", Carbon 45 (2007) 1453-1462; doi:10.1016/j.carbon.2007.03.028.

[9] B. J. Cox and J. M. Hill, "A variational approach to the perpendicular joining of nanotubes to plane sheets", J. Phys. A: Math. Theor. 41 (2008) 125203; doi:10.1088/1751-8113/41/12/125203.

[10] F. Ding, Y. Lin, P. O. Krasnov and B. I. Yakobson, "Nanotube-derived carbon foam for hydrogen sorption", J. Chem. Phys. 127 (2007) doi:10.1063/1.2790434.

[11] M. S. Dresselhaus, G. Dresselhaus and P. C. Eklund, Science of fullerenes and carbon nanotubes (Academic Press, San Diego, 1995).

[12] M. S. Dresselhaus, G. Dresselhaus and R. Saito, "Physics of carbon nanotubes", Carbon 33 (1995) 883-891; doi:10.1016/0008-6223(95)00017-8.

[13] M. Ge and K. Sattler, "Observation of fullerene cones", Chem. Phys. Lett. 220 (1994) 192-196; doi:10.1016/0009-2614(94)00167-7.

[14] A. Krishnan, E. Dujardin, M. M. J. Treacy, J. Hugdahl, S. Lynum and T. W. Ebbesen, "Graphitic cones and the nucleation of curved carbon surfaces", Nature 388 (1997) 451-454; doi: $10.1038 / 41284$.

[15] A. G. Nasibulin et al., "A novel hybrid carbon material", Nature Nanotech. 2 (2007) 156-161; doi:10.1038/nnano.2007.37.

[16] R. Pincak and V. A. Osipov, "Localized electron states near pentagons in variously shaped carbon nanoparticles", Phys. Lett. A 314 (2003) 315-321; doi:10.1016/S0375-9601(03)00898-3.

[17] R. Saito, G. Dresselhaus and M. S. Dresselhaus, "Tunneling conductance of connected carbon nanotubes", Phys. Rev. B 53 (1996) 2044-2050; doi:10.1103/PhysRevB.53.2044.

[18] R. Saito, G. Dresselhaus and M. S. Dresselhaus, Physical properties of carbon nanotubes (Imperial College Press, London, 1998).

[19] K. Sattler, "Scanning tunneling microscopy of carbon nanotubes and nanocones", Carbon 33 (1995) 915-920; doi:10.1016/0008-6223(95)00020-E.

[20] H. Ueno, S. Osawa, E. Osawa and K. Takeuchi, "Stone-Wales rearrangement pathways from the hinge-opened [2+2] $\mathrm{C}_{60}$ dimer to IPR $\mathrm{C}_{120}$ fullerenes. Vibrational analysis of intermediates", Fullerene Sci. Tech. 6 (1996) 319-338.

[21] Y. Xia, Y. Xing, C. Tan and L. Mei, "Dimerization and fusion of $\mathrm{C}_{60}$ molecules caused by molecular collision", Phys. Rev. B 53 (1996) 13871-13876; doi:10.1103/PhysRevB.53.13871.

[22] Y. Xie, J. Huang, B. Li, Y. Liu and Y. Qian, "A novel peanut-like nanostructure of II-VI semiconductor CdS and ZnS", Adv. Mater. 12 (2000) 1523-1526; doi:10.1002/1521-4095(200010)12:20<1523::AID-ADMA1523>3.3.CO;2-K.

[23] J. Zang, A. Treibergs, Y. Han and F. Liu, "Geometric constant defining shape transitions of carbon nanotubes under pressure", Phys. Rev. Lett. 92 (2004) doi:10.1103/PhysRevLett.92.105501.

[24] Y. Zhao, Y. Lin and B. I. Yakobson, "Fullerene shape transformations via Stone-Wales bond rotations", Phys. Rev. B 68 (2003) doi:10.1103/PhysRevB.68.233403.

[25] Y. Zhao, R. E. Smalley and B. I. Yakobson, "Coalescence of fullerene cages: topology, energetics, and molecular dynamics simulation", Phys. Rev. B 66 (2002) doi:10.1103/PhysRevB.66.195409.

[26] Y. Zhao, B. I. Yakobson and R. E. Smalley, "Dynamic topology of fullerene coalescence", Phys. Rev. Lett. 88 (2002) doi:10.1103/PhysRevLett.88.185501. 